\title{
Schwa-Elision und die Wortprosodie des Luxemburgischen
}

\author{
François Conrad (Hannover)
}

\begin{abstract}
In the young Germanic language Luxembourgish schwa can be dropped in the context before (and sometimes after) a sonorant and following a stressed syllable, e. g. Elteren $>$ Elt_ren 'parents'. The present paper describes schwa deletion from three perspectives: (1) the influence of speech rate (allegro vs. lento speech), (2) the influence of the segments adjacent to schwa and (3) the prosodic structure of words. The results of an empirical investigation with 15 native speakers show that the deletion is very frequent in spoken Luxembourgish and that the two first aspects jointly account for the phenomenon. Regarding the third aspect, due to the loss of the vowel and hence of a syllable the predominance of the dactyl shifts towards the trochee, which constitutes the unmarked foot type in spoken Luxembourgish.
\end{abstract}

\section{$1 \quad$ Einleitung}

Im gesprochenen Luxemburgischen (Lb.) - einer Sprache, die sich seit einigen Jahrzehnten aus dem Gefüge der angrenzenden moselfränkischen Dialekte löst (Gilles 1998) - wird der Schwa-Vokal in Wörtern, in denen eine unbetonte Schwasilbe auf eine betonte Silbe folgt, häufig getilgt: Aus d'Duechtere bastelen ,die Töchter basteln' wird d'Duecht_re bast_len. ${ }^{1}$

Vorliegende Arbeit untersucht die Gesetzmäßigkeiten des Ausfalls dieses im Lb. sehr häufigen Vokals. Neben den Funktionen eines betonbaren Vollvokals (etwa in Blëtz [bləts] ,Blitz') erfüllt er ebenfalls Funktionen eines Reduktionsvokals, die denen des Deutschen (Dt.) entsprechen. Nach der phonologischen Situierung des Phänomens (Punkt 2) und der Darstellung des empirischen Erhebungsverfahrens (3) bespricht die Arbeit den Einfluss der Redegeschwindigkeit (der realisationsphonetische Aspekt; Punkt 4) und denjenigen der benachbarten Segmente (der artikulatorische Aspekt; Punkt 5) auf die Häufigkeit der Elision sowie die weitreichenden Konsequenzen des Ausfalls auf die prosodische Struktur der lb. Substantive, Adjektive und Verben (6).

- In Anlehnung an die deutschsprachige Literatur wird das Phänomen im Folgenden als Schwa-Elision bezeichnet. ${ }^{2}$ Alternativ wird der Begriff Schwa-Ausfall verwendet.

\footnotetext{
${ }^{1}$ Der vorliegende Artikel ist eine umstrukturierte Zusammenfassung der Magisterarbeit von Conrad (2010) an den Universitäten Bamberg und Luxemburg. Für wertvolle Hinweise danke ich Uta Reinöhl, Cristian Kollmann, Caroline Döhmer und drei anonymen GutachterInnen. Den SprecherInnen, die an dem Sprachexperiment teilgenommen haben, danke ich sehr für Ihre Mitarbeit.

2 Etwa bei Kohler $(1979,1990,1995)$ und Meinhold/Stock (1982). Als Alternativen finden sich z. B. Tilgung bei Pompino-Marschall (2009: 274), Vokalverlust bei Dressler (1972a: 58) und Synkope bei Nübling (2005).

Linguistik online 80, 1/17 - http://dx.doi.org/10.13092/lo.80.3564
}

CC by 3.0 
- Als junge germanische Sprache ist das Lb. phonologisch noch wenig erforscht. Aus diesem Grund wird insbesondere das Dt. (vereinzelt auch das Niederländische und das amerikanische Englisch) als Vergleichsbasis herangezogen.

\section{Die Schwa-Elision im Deutschen, Niederländischen und Luxemburgischen}

Der Ausfall des unbetonten Schwa-Vokals ist u. a. auch im Dt. und im Niederländischen (Ndl.) bezeugt. ${ }^{3}$ Im Dt. ist laut Kohler (1979: 17) vor allem ,die unakzentuierte Silbe unmittelbar nach dem Akzent [betroffen] [...], stärker als die Silbe vor dem Akzent und stärker als die weiter entfernte Silbe nach dem Akzent". Diese unbetonte, dem Akzent unmittelbar folgende Schwa-Silbe ist in zwei Kontexten besonders elisionsanfällig: a) Schwa vor Sonorant in der einzigen Schwa-Silbe des Wortes (e. g. Segel [ze:g!]) und b) Schwa in der Umgebung von Sonorant in der ersten von zwei aufeinanderfolgenden Schwa-Silben.

Kontext a) betrifft die Verbindungen -en, -em, -el, und -er. Wird Schwa elidiert, übernimmt der Sonorant in vielen Fällen silbische Funktion. Kontext b) umfasst viele flektierte Adjektive und Substantive sowie konjugierte Verben. In diesen Fällen ist die Elision zumeist optional, e. g. bess[ə]rer, Wand[ə]rer, verbot[a]ne (Beispiele aus Kohler 1990: 84). Beide Formen stehen „fakultativ zur Verfügung, je nach Sprechgeschwindigkeit, rhythmischer Umgebung und Belieben“" (Meinhold/Stock 1982: 201).

Auch das Ndl. kennt das Phänomen. Unter schwa deletion schreibt etwa Booij:

When a Dutch word has two consecutive syllables headed by a schwa, the first of these schwas may be deleted, provided that the resulting onset consonant cluster be an obstruent + liquid cluster, universally the most favored type of onset cluster.

(1995: 128)

Es handelt sich also um den für das Dt. postulierten Kontext b) mit der Einschränkung, dass vor Schwa ein Obstruent, nach Schwa ein Liquid stehen muss. ${ }^{4}$ Wie im Dt. ist die ə-Elision fakultativ („may be deleted“) und es gibt ein Nebeneinander von Formen mit und ohne Schwa, etwa in off[ə]ren ,opfern'. Erneut ist das Schwa nach dem akzentuierten Vokal betroffen.

In der lb. Forschungsliteratur findet die Schwa-Elision kaum Erwähnung. ${ }^{5}$ Ein Punkt, den einige Forscher jedoch hervorheben (etwa Gilles 1998, 2014; Nübling 2005; Krier 2008) ist die „Synkoperesistenz“: Insbesondere in der Verbalendung -en wird das Schwa stets realisiert und es findet keine Elision - und somit auch keine Silbizität der Sonoranten - statt. $^{6}$ Im Lb. fällt der dt. Kontext a) also aus.

\footnotetext{
${ }^{3}$ Ausführlich zur Phonologie des Schwa-Vokals im Deutschen etwa bei Wiese (1996). Die dort und allgemein häufig besprochene Schwa/Null-Alternation vom Typ Handel vs. Handlung stellt ein von den untersuchten Daten in vorliegender Arbeit unterschiedliches Phänomen dar.

${ }^{4}$ Bsp. bei Booij (1995: 129; Auswahl): wisselen [wislən] ,wechseln‘, kegelen [ke:ylən] ,kegeln“, offeren [ofrən] ,opfern“. Auf den deutschen Kontext a) geht Booij für das Nied. nicht ein. Booij (ibd.: 130) legt dem Phänomen artikulatorische Ursachen zugrunde: ,The rule decreases the articulatory effort required for the pronunciation of the relevant words in that there is one syllable less to produce“".

${ }^{5}$ Eine Ausnahme stellt Gilles (2014) dar, der sich jedoch insbesondere auf Conrad (2010) beruft. Er bespricht die Schwa-Elision im Rahmen der Silben-/Wortsprachlichkeit des Lb. und deutet sie als wortsprachliches Phänomen.

${ }^{6}$ Gilles (1998: 32) spricht allgemein vom „Ausbuchstabieren von Schwa in unbetonten Silben“, bezieht sich in seinen Beispielen aber vor allem auf die Verben (er nennt u. a. wiesselen [viəsələn] ,wechseln', baken [ba:kən]
} 
Kontext b) wird etwa bei Schanen/Zimmer (2006b: 51-52) erwähnt: Innerhalb eines Wortes kann Schwa vor den Sonoranten $r, l, m, n$ in einer schnelleren Aussprache (,langue plus flui$\mathrm{de}^{\text {") }}$ ) elidiert werden (Schanen/Zimmer benutzen das Wort ,assimiler"), wenn eine weitere Silbe folgt. Als Beispiele nennt er u. a. ma'kab(e)ren ,makaberer', e 'propp(e)ren Auto ,ein sauberes Auto', 'krabb(e)len ,krabbeln', 'héi(e)ren ,hören' und en 'donk(e)le Kostüm ,ein dunkler Anzug'. ${ }^{7}$

Auch im Lb. ist also stets ein Sonorant involviert. Dieser kann wie im Dt. (und entgegen dem Ndl.) auch ein Nasal sein, und entgegen Schanen/Zimmer selbst vor Schwa stehen, etwa in hell(e)gen ,heiliger‘. Die Optionalität der Elision wird im Titel des Kapitels bei Schanen/Zimmer hervorgehoben („Le [ə] syllabisé facultatif en syllabe non-accentuée“; vgl. auch Krier [2008: 104], die von „freien Varianten“ spricht). Für alle Fälle gilt, dass die volle Form die schriftsprachliche Norm darstellt.

Das Phänomen der ə-Elision ist folglich in allen drei benachbarten germanischen Sprachen präsent, unterscheidet sich jedoch leicht in der Ausprägung. Tabelle 1 stellt die Kontexte in der jeweiligen Sprache (vereinfacht) zusammen.

\begin{tabular}{|l|l|l|}
\hline Kontext & $\begin{array}{l}\text { a) ə vor Son. (einzige } \\
\text { Schwa-Silbe im Wort) }\end{array}$ & $\begin{array}{l}\text { b) Schwa-Silbe nach Akzent und vor } \\
\text { weiterer Silbe }\end{array}$ \\
\hline & -en/-em/-el/-er & Schwa vor/nach Son. \\
\hline Deutsch & & fakultativ \\
\hline Elision & Ja & Nein \\
\hline Silbizität des Son. & Ja & fakultativ \\
\hline Niederländisch & & Nein \\
\hline Elision & Nein & fakultativ \\
\hline Silbizität des Son. & Nein & Nein \\
\hline Luxemburgisch & & Nein \\
\hline Elision & Nein & S \\
\hline Silbizität des Son. &
\end{tabular}

Tabelle 1: Kontexte der ə-Elision im Dt., Ndl. und Lb. $($ vereinfacht $)($ Son. = Sonorant; Ja = möglich; Nein $=$ nicht möglich)

Nur Kontext b) gilt für alle drei germanischen Sprachen. Kontext a) tritt nur im Dt. auf. In allen Fällen ist unbetontes Schwa in der Umgebung von Sonoranten betroffen. Dieses steht stets in der Silbe, die der betonten Silbe unmittelbar folgt.

Neben dem wichtigen Aspekt der phonologischen Umgebung des Schwa-Vokals (Kohler 1979, 1995; Booij 1995; Schanen/Zimmer 2006b) wurden auch der Artikulationsaufwand und die damit verbundene Sprechgeschwindigkeit (Meinhold/Stock 1982; Booij 1995) erwähnt. Um den Einfluss dieser Aspekte auf die Schwa-Elision im Lb. festzustellen, wurden zahlreiche Wörter, die obigen Bedingungen entsprechen (Schwa-Silbe nach betonter Silbe, sonoran-

,backen“ und schwammen [Jwamon] ,schwimmen“). Die Auswirkungen dieser „Synkoperesistenz“ auf die lb. Prosodie werden unter 6.4 besprochen.

${ }^{7} \mathrm{Im} \mathrm{Lb}$. gibt es noch einen dritten Fall von ə-Elision in Wörtern mit anlautendem Schwa-Vokal (Schanen/Zimmer 2006b: 51), etwa (e)lei ,hier', (e)lo ,jetzt', (e)sou ,so', (e)rof ,(he)rab', (e)wech ,weg'. Dieser spielt in vorliegender Untersuchung jedoch keine Rolle. 
tisches Umfeld) experimentell untersucht. Das nächste Kapitel stellt das Design dieses Experiments vor.

\section{$3 \quad$ Methodologie der empirischen Untersuchung}

\subsection{Korpus}

Für die Untersuchung der Schwa-Elision und deren Faktoren konnte auf kein bestehendes lb. Korpus zurückgegriffen werden. Es wurden daher eigene Spracherhebungen durchgeführt. Dies hat den großen Vorteil, dass das Untersuchungsdesign sowie die Wahl der SprecherInnen dem Untersuchungsgegenstand angepasst werden konnten.

Trotz seiner kleinen Fläche weist das Großherzogtum eine hohe Dialektvielfalt auf (Gilles 1999). Die vorliegende Untersuchung wurde auf die zentrallb. Varietät beschränkt. ${ }^{8}$ Es wurden für die Aufnahmen nur SpecherInnen gewählt, die im Kerngebiet dieses Dialektraums (Luxemburg-Stadt und Umgebung, siehe Gilles 1999: 62) aufgewachsen sind - der Großteil von ihnen wohnt auch heute noch dort - und die entsprechende Varietät auch im Elternhaus gesprochen haben. Alle SprecherInnnen haben die lb. Staatsbürgerschaft und Lb. als Muttersprache. Es handelt sich somit um eine dialektal sehr homogene Gruppe.

Die SprecherInnen wurden in drei Generationen eingeteilt, die grob einem Generationssprung entsprechen. Diese Vorgehensweise erlaubt Einblicke in altersbedingte Unterschiede sowie mögliche sprachliche Entwicklungen. Tabelle 2 zeigt die Einteilung der 15 SprecherInnen der Untersuchung:

\begin{tabular}{|l|l|l|}
\hline Bezeichnung der Generation & Alter & Anzahl SprecherInnen \\
\hline Kinder & 15-30 Jahre & $5(3 \mathrm{w}, 2 \mathrm{~m})$ \\
\hline Eltern & 31-60 Jahre & $5(3 \mathrm{w}, 2 \mathrm{~m})$ \\
\hline Großeltern & 61-100 Jahre & $5(2 \mathrm{w}, 3 \mathrm{~m})$ \\
\hline
\end{tabular}

Tabelle 2: Die Einteilung der SprecherInnen im Sprachexperiment

\subsection{Der Aufbau des Experiments}

Insgesamt wurden 89 Testwörter untersucht. Anhand einer am Computer erstellten Bildschirmpräsentation wurden den SprecherInnen Bilder gezeigt, unter denen einzelne Wörter, Wortketten oder ganze Sätze standen. Vereinzelt traten auch französische (fr.) Wörter auf, die ins Lb. zu übersetzen waren. Um einen möglichen Einfluss der Schrift auszuschließen, wurde die Silbe mit elisionsfähigem Schwa-Vokal nicht ausbuchstabiert, sondern erschien als Lücke (siehe Anhang $b$ für Beispiele). ${ }^{9}$ Die Aufgabe bestand für die SprecherInnen darin, die nacheinander am Computerbildschirm erscheinenden Wörter/Sätze in zwei unterschiedlichen Geschwindigkeiten (ausführlich hierzu unter Punkt 4.2) zu äußern. Die Äußerungen wurden mit einem Mikrofon (Sennheiser HSP 2) aufgezeichnet.

\footnotetext{
${ }^{8}$ Diese stellt Zentrum und Motor des fortschreitenden Dialektausgleiches dar (Gilles 1999).

${ }^{9}$ Um die Aufmerksamkeit der SprecherInnen nicht auf diese Silben zu lenken, wurde die Lücke häufig auf das gesamte Wortende ausgedehnt. Zusätzlich wurden einige Distraktoren eingebaut, bei denen Wörter zu sprechen waren, die für die Untersuchung keine Rolle spielten.
} 
Im ersten Teil sollten sie möglichst natürlich und ohne bewusste Artikulation sprechen (Allegro). Er beinhaltete überwiegend ganze Sätze, etwa

De Max ass en fait e mont(ere) Kärel a war schonn ëmmer e sensi(bele) Jong.

,Max ist eigentlich ein munterer Kerl und war schon immer ein sensibler Junge.

Die Wörter en fait waren aus dem Fr. zu übersetzen und entsprechend markiert.

Im zweiten Teil war die Vorgabe zu sprechen, als würde man einem Lb.-Lernenden die richtige Aussprache zeigen: deutlich, langsam und bewusst, ohne aber unnatürlich zu klingen (Lento). In diesem Teil traten nur Wörter oder kleine Wortketten auf, etwa e mont(ere) Bouf ,ein munterer Junge'.

Der Großteil der Stimuli $(\mathrm{N}=33)$ erschien sowohl im ersten als auch im zweiten Teil des Experiments. Hierdurch konnte der Einfluss der unterschiedlichen Redegeschwindigkeiten auf den gleichen Stimulus untersucht werden (realisationsphonetischer Aspekt). Die Wörter wurden zudem so ausgewählt, dass verschiedene Wortarten vertreten waren (zur Untersuchung der Wortprosodie) und der Schwa-Vokal in unterschiedlicher phonologischer Umgebung stand (artikulatorischer Aspekt). Die drei Aspekte konnten somit anhand des gleichen Experiments untersucht werden.

Bei der Auswertung, die auditiv erfolgte, wurde für jedes Wort festgehalten, ob der elisionsfähige Schwa-Vokal artikuliert wurde. In einigen Fällen war nicht klar zu erkennen, ob ein Schwa vorhanden war oder nicht. Alle diese Fälle wurden als „unklar“ markiert. Neben ,elidiert“ und ,nicht-elidiert“ entstand so eine dritte Kategorie. Diese floss in die Ergebnisse mit ein, da der Prozentsatz an unklaren Fällen Rückschlüsse auf die Artikulationsgenauigkeit bestimmter Verbindungen erlaubt.

Da die Darstellung der Ergebnisse in den weiteren Punkten zumeist auch die unterschiedlichen Redegeschwindigkeiten abbildet, wird im folgenden Punkt zunächst der Einfluss dieser Geschwindigkeit auf die Elision besprochen. Im Anschluss werden die artikulatorischen und prosodischen Aspekte beleuchtet.

\section{$4 \quad$ Sprechgeschwindigkeit als Steuerungsfaktor der Schwa-Elision}

\subsection{Der Einfluss der Sprechgeschwindigkeit auf die Artikulation}

Eine Kategorisierung verschiedener Sprechtempora, wie sie für diese Untersuchung notwendig ist, ist nicht einfach. Die vielfältigen Geschwindigkeiten und Begriffe werden in der Forschung unterschiedlich verwendet und sind ,großen Schwankungen und Variationen unterworfen“ (Dressler 1972b: 4). In Anlehnung an die lb. Forschung (Gilles 1998, 1999; Schanen 2006) werde ich zwischen einer langsamen, bewussten Sprechweise (Lento) und einer schnelleren, unbewussteren Sprechweise (Allegro) unterscheiden. ${ }^{10}$ Ihre Auslegung, wie sie Tabelle 3 darstellt, ist den Erfordernissen des Untersuchungsdesigns und -gegenstands angepasst.

\footnotetext{
${ }^{10}$ Die Ausprägung von Lento und Allegro variierte sprecherindividuell: Es konnte durchaus vorkommen, dass rein an der Geschwindigkeit gemessen - der Allegro-Stil der einen Person dem Lento-Stil einer anderen Person entsprach. In allen Fällen unterschieden sich jedoch Allegro und Lento innerhalb derselben Person, so dass die sprecherindividuellen Differenzen beim Sprachexperiment keine Rolle spielten.
} 


\begin{tabular}{|l|l|l|l|l|l|}
\hline $\begin{array}{l}\text { Bezeich- } \\
\text { nung }\end{array}$ & $\begin{array}{l}\text { Geschwin- } \\
\text { digkeit }\end{array}$ & Artikulation & $\begin{array}{l}\text { Aufmerk- } \\
\text { samkeit } \\
\text { gerichtet } \\
\text { auf }\end{array}$ & Äußerung & Entsprechung \\
\hline Lento & langsam & $\begin{array}{l}\text { bewusst, alle Lau- } \\
\text { te werden artiku- } \\
\text { liert, aber keine } \\
\text { Hyperartikulation }\end{array}$ & Artikulation & $\begin{array}{l}\text { Wörter oder } \\
\text { kleine } \\
\text { Wortketten }\end{array}$ & $\begin{array}{l}\text { geschriebene } \\
\text { Sprache, Wör- } \\
\text { terbucheintrag }\end{array}$ \\
\hline Allegro & $\begin{array}{l}\text { Gesprächs- } \\
\text { ge- } \\
\text { schwindig- } \\
\text { keit }\end{array}$ & $\begin{array}{l}\text { unbewusst, es } \\
\text { kann zu Redukti- } \\
\text { onen kommen }\end{array}$ & Inhalt & $\begin{array}{l}\text { Wörter im } \\
\text { Satzzu- } \\
\text { sammen- } \\
\text { hang }\end{array}$ & $\begin{array}{l}\text { gesprochene } \\
\text { Sprache, All- } \\
\text { tagssprache }\end{array}$ \\
\hline
\end{tabular}

Tabelle 3: Parameter von „Lento“ und „Allegro“ für das Sprachexperiment

Aus den Darlegungen der Punkte 4.1 und 4.2 ergibt sich Hypothese 1 (Hyp. 1):

- Hypothese 1: Erhöhte Sprechgeschwindigkeit begünstigt die Schwa-Elision im Luxemburgischen.

Die Elision wäre laut Hyp. 1 häufiger in den im Allegro-Stil geäußerten Wörtern als in denjenigen, die im Lento-Stil gesprochen werden. Treten elidierte Fälle in beiden Geschwindigkeiten etwa gleich oft auf oder überwiegen die Fälle im Lento, muss Hyp. 1 verworfen werden.

Um Hyp. 1 zu prüfen, wurden insgesamt 89 Wörter ins Experiment eingebaut, die einen nach den unter Punkt 2 dargelegten Bedingungen elisionsfähigen Schwa-Vokal aufweisen (Kontext vor/nach Sonorant, Nukleus der Silbe, die dem Wortakzent unmittelbar folgt), etwa Konf(e)renz, Tromm(e)ler. 36 Wörter sollten dabei in Lento, 54 Wörter in Allegro gesprochen werden $(\mathrm{AW}=$ Alle Wörter). 33 Wörter hiervon traten sowohl im Lento- als auch im AllegroTeil des Experiments auf und konnten direkt miteinander verglichen werden $(\mathrm{GW}=$ Gleiche Wörter). ${ }^{11}$ Anhang $a$ listet die einzelnen Wörter auf. ${ }^{12}$

\footnotetext{
${ }^{11}$ Daneben kamen drei Wörter vor, die nur im Lento-Teil, sowie 21 Wörter, die nur im Allegro-Teil auftraten. Diese Wörter konnten aufgrund der kleinen Größe des jeweiligen Korpus nicht direkt miteinander verglichen werden, wurden aber in die Summe der Fälle von (Nicht-)Elision mit einbezogen.

12 Jeder Stimulus wurde zweimal hintereinander gesprochen. Diese beiden Aussprachen unterschieden sich jedoch zu weniger als $5 \%$, so dass dies für die weitere Analyse keine Rolle spielt.
} 


\subsubsection{SprecherInnen gesamt}

Tabelle 4 zeigt das Gesamtergebnis der 15 SprecherInnen. Die Daten sind in Prozent (abgerundet) angegeben. Schwa-Elision tritt bei jenen Wörtern, die sowohl im Lento als auch im Allegro auftreten, zu $71 \%$, im Lento hingegen nur zu $36 \%$ auf. Die Differenz beträgt $35 \%$. Nimmt man die Fälle hinzu, die entweder nur im Lento oder nur im Allegro vorlagen, ändern sich die Zahlen nur leicht: Der Prozentsatz elidierter Fälle im Allegro sinkt auf 64 \%, derjenige im Lento steigt hingegen leicht auf $40 \%$, bei einer Differenz von $24 \%$.

Die Prozentsätze der Fälle ohne Schwa-Elision unterscheiden sich entsprechend: Zeigen $53 \%$ (GW) bzw. 48 \% (AW) der Fälle im Lento volle Formen, sind es im Allegro nur $20 \%$ (GW) bzw. $24 \%$ (AW). Im Lento-Stil werden also wesentlich mehr ,volle“ Formen realisiert als im Allegro-Stil. Etwa 1/10 aller Wörter wurde als ,unklar“ eingestuft. ${ }^{13}$

\begin{tabular}{|l|c|c|c|}
\hline \multirow{2}{*}{} & \multicolumn{3}{|l|}{ Gleiche Wörter Allegro + Lento (GW) } \\
& Lento & Allegro & Differenz \\
\hline Elidiert & $36 \%$ & $\mathbf{7 1} \%$ & $35 \%$ \\
\hline nicht-elidiert & $\mathbf{5 3 \%}$ & $20 \%$ & $-33 \%$ \\
\hline Unklar & $11 \%$ & $9 \%$ & $-2 \%$ \\
\hline Gesamt & $100 \%$ & $100 \%$ & $0 \%$ \\
\hline
\end{tabular}

\begin{tabular}{|l|c|c|}
\hline \multicolumn{3}{|l|}{ Alle Wörter (AW) } \\
\hline Lento & Allegro & Differenz \\
\hline $40 \%$ & $\mathbf{6 4} \%$ & $24 \%$ \\
\hline $48 \%$ & $24 \%$ & $-24 \%$ \\
\hline $12 \%$ & $12 \%$ & $0 \%$ \\
\hline $100 \%$ & $100 \%$ & $0 \%$ \\
\hline
\end{tabular}

Tabelle 4: Ergebnisse SprecherInnen gesamt; Werte von mind. 50 \% stehen in Fettdruck

Insgesamt lässt sich Hyp. 1 anhand der Werte in Tabelle 4 also bestätigen. Insbesondere bei der direkten Gegenüberstellung der gleichen Wörter (GW) fallen die Elisionswerte im Allegro höher aus als im Lento. Diese Unterschiede sind auch statistisch signifikant. ${ }^{14}$ Steigende Sprechgeschwindigkeit begünstigt also die Schwa-Elision. Mit Elisionswerten von $40 \%$ auch im Lento kann das Phänomen als im Lb. allgemein sehr häufig angesehen werden.

\subsubsection{Die einzelnen SprecherInnen}

In einem nächsten Schritt werden die Werte der einzelnen SprecherInnen vergleichend gegenübergestellt. Dadurch soll v. a. gezeigt werden, ob bestimmte SprecherInnen aufgrund „extremer“ Werte die Gesamtergebnisse beeinflussen. Für die direkte Gegenüberstellung werden nur die Gesamtwerte (AW) gezeigt, für Allegro, Lento und die unklaren Fälle.

\footnotetext{
13 Diese hohe Zahl hat artikulationstechnische Gründe (siehe Punkt 5). Entgegen meiner Vermutung vor der Auswertung der Daten zeigt sich keine höhere Zahl unklarer Fälle im Allegro als im Lento. Die Redegeschwindigkeit ist also nicht für die unklaren Fälle verantwortlich.

${ }^{14} \mathrm{Da}$ die Elisionshäufigkeiten pro Person mitunter dreistellig ausfallen, werden diese für jede Person und jede Redegeschwindigkeit gemittelt. Anhand eines $t$-Tests können diese gemittelten Werte gegenübergestellt werden. Abhängiger $t$-Test; GW, Allegro vs. Lento: Elision: $t(14)=13,67, \mathrm{p}<0,001$; keine Elision: $t(14)=-16,98$, $\mathrm{p}<0,001$; AW, Allegro vs. Lento: Elision: $t(14)=9,31, \mathrm{p}<0,001$; keine Elision: $t(14)=-8,95, \mathrm{p}<0,001$.
}

ISSN 1615-3014 


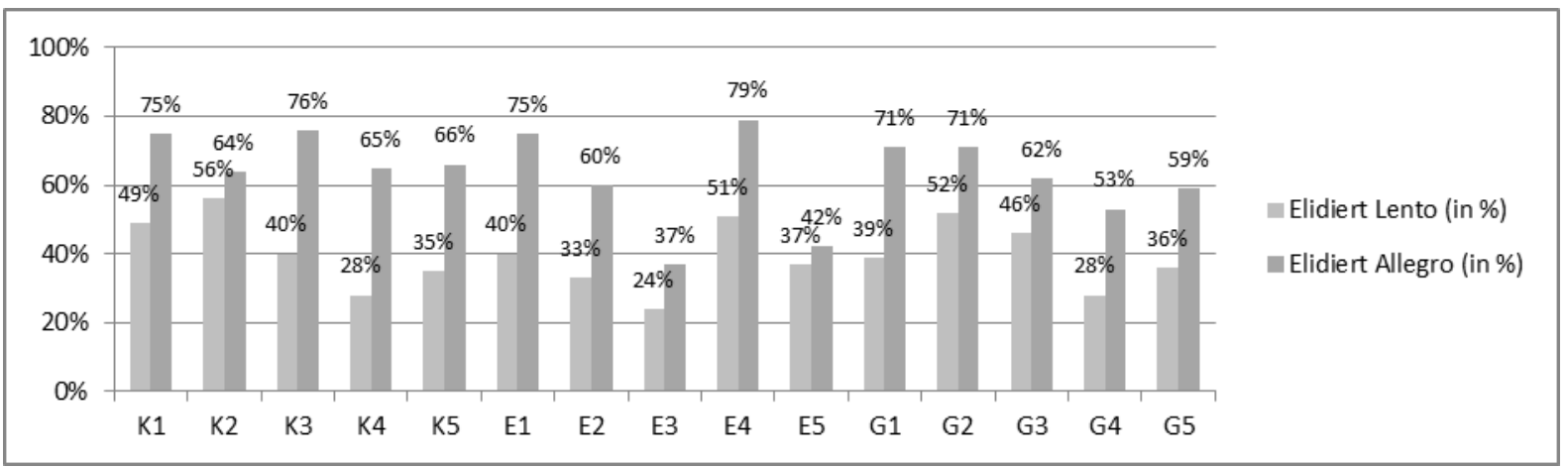

Abbildung 1: Häufigkeit von Schwa-Elision der einzelnen SprecherInnen im Lento und Allegro (in \%). Schnitt Allegro: 64 \%, Schnitt Lento: 40 \% (K = Generation „Kinder“, E = Generation „Eltern“, G = Generation ,Großeltern“6)

Für die Anzahl an elidierten Formen zeigt Abbildung 1, dass der Streubereich sowohl im Lento als auch im Allegro sehr groß ist. Die Zahl variiert im Lento zwischen $24 \%$ (E3) und $56 \%$ (K2) (Differenz von $32 \%$ ), im Allegro zwischen $37 \%$ (E3) und $79 \%$ (E4) (Differenz von $42 \%)$.

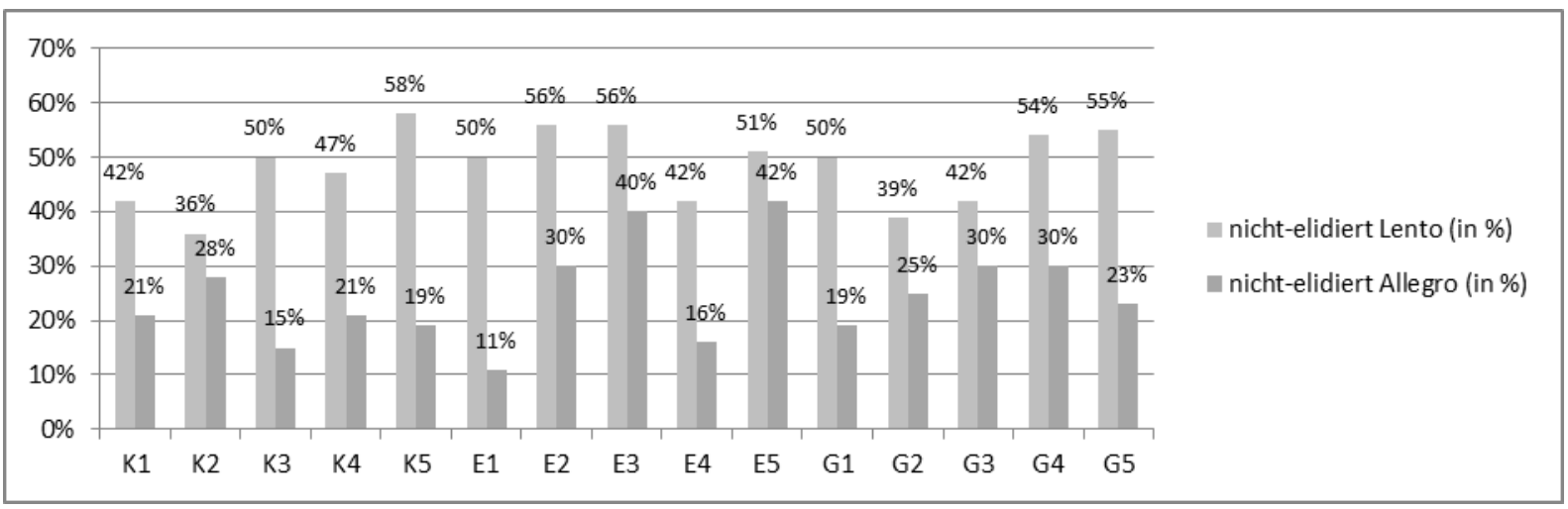

Abbildung 2: Häufigkeit voller Formen der einzelnen SprecherInnen im Lento und Allegro (in \%). Schnitt Allegro: $25 \%$, Schnitt Lento: 48 \% (K= Generation „Kinder“" E = Generation „Eltern“, G = Generation „Großeltern“")

Auch für die nicht-elidierten Fälle zeigt sich ein großer Streubereich, wenngleich dieser weniger stark ausgeprägt ist. Er reicht im Lento von $36 \%$ (K2) bis $58 \%$ (K5) (Differenz von $22 \%$ ). Die Fälle im Allegro zeigen insgesamt niedrigere Prozentzahlen und reichen von $11 \%$ (E1) bis $42 \%$ (E5) (Differenz von $31 \%$ ).

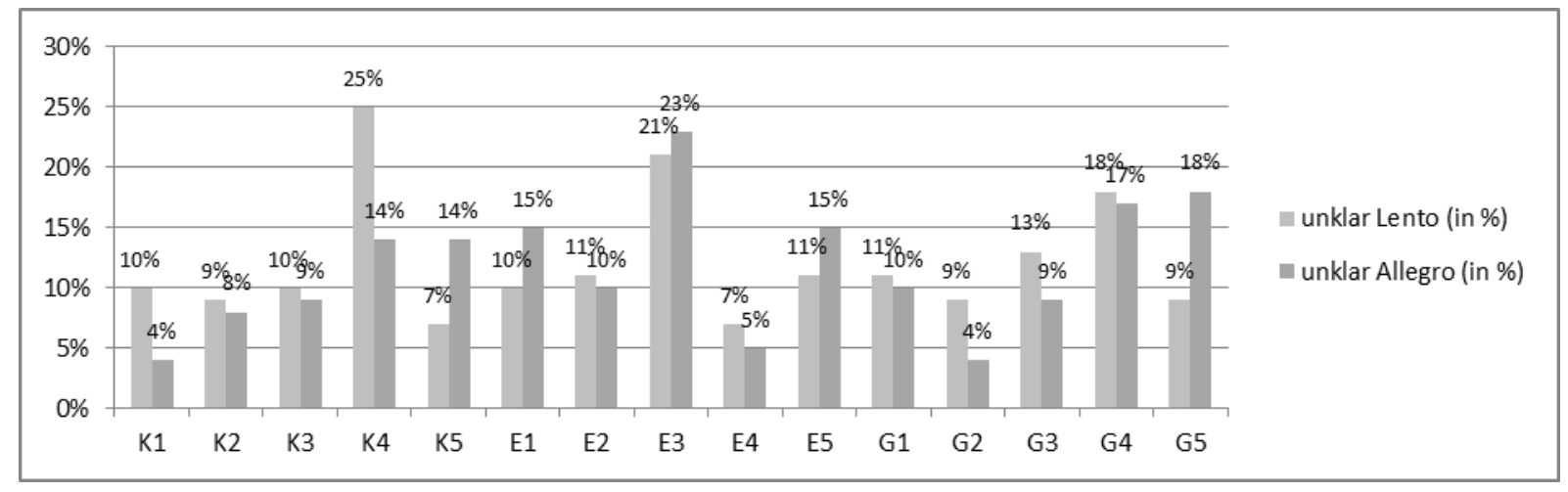

Abbildung 3: Unklare Fälle der einzelnen SprecherInnen im Lento und Allegro (in \%). Schnitt Allegro: $12 \%$, Schnitt Lento: $12 \%$ (K = Generation „Kinder“, E = Generation „Eltern“, G = Generation „Großeltern") 
Auch bei den unklaren Fällen zeigen sich teils große Unterschiede zwischen den SprecherInnen. Im Lento liegen die Minima/Maxima bei $7 \%$ (K5 und E4) bzw. $25 \%$ (K4) (Differenz von $18 \%$ ), im Allegro bei $4 \%$ (K1 und G2) bzw. $23 \%$ (E3) (Differenz von 19\%).

Die Daten in den Abbildungen 1-3 weisen also allesamt eine große Varianz zwischen den verschiedenen SprecherInnen auf. ${ }^{15}$ Sind die Durchschnittswerte für die Schwa-Elision im Allegro insgesamt sehr hoch, finden sich unter den SprecherInnen sowohl solche mit extrem hohen als auch solche mit vergleichsweise niedrigen Werten.

Für die Besprechung von Hyp. 1 (Erhöhte Sprechgeschwindigkeit begünstigt die SchwaElision im Luxemburgischen) sind nun die Unterschiede besonders relevant, die bei den einzelnen SprecherInnen zwischen dem Prozentsatz elidierter Formen im Lento und Allegro bestehen (Abb. 4).

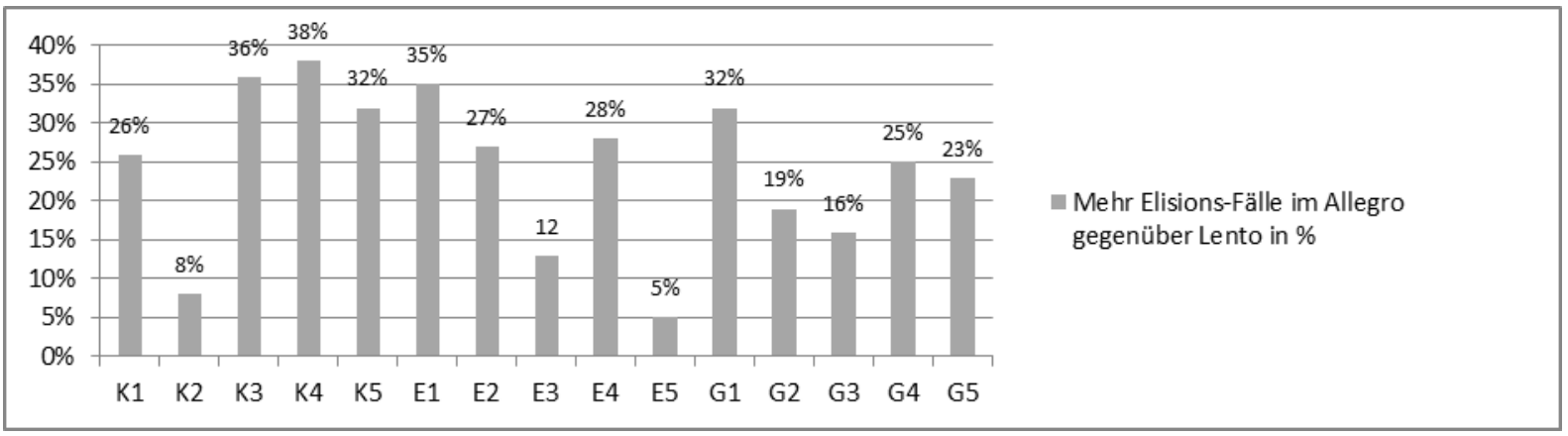

Abbildung 4: Größere Zahl an Fällen mit Schwa-Elision im Allegro gegenüber Lento (in \%) (K= Generation ,Kinder", E = Generation „Eltern“, G = Generation „Großeltern“")

Auch die Daten in Abbildung 4 zeigen einen großen Streubereich zwischen $38 \%$ (K4) und $5 \%$ (E5) (Differenz von $33 \%$ ). 10 von 15 SprecherInnen zeigen allerdings im Allegro mindestens 23 \% mehr Fälle von Elision auf als im Lento (bei nur zwei Fällen unter $10 \%$ ). Eine schnellere Sprechweise ruft also bei jeder Versuchsperson (teils deutlich) mehr Fälle von Schwa-Elision hervor als eine langsamere. Die Unterschiede können folglich nicht auf einzelne, besonders häufig elidierende Personen zurückgeführt werden.

Was die Differenzen zwischen den Generationen angeht, zeigt lediglich die Generation „Kinder" leicht erhöhte Elision im Allegro gegenüber Lento im Vergleich mit den anderen Generationen („Kinder“: $28 \%$, „Eltern“: $21 \%$, „Großeltern“: $23 \%$ ). Dieser Unterschied ist allerdings statistisch nicht signifikant. ${ }^{16}$ Auch die Geschlechter unterscheiden sich nicht signifikant voneinander (Männer: $22 \%$, Frauen: $26 \%$; $t$-Test: $t(13)=-0,83$, n. s.).

\subsection{Schlussfolgerung}

Die Auswertung der Sprachdaten von 15 Personen, die allesamt Wörter mit potenziellem Schwa-Ausfall in zwei unterschiedlichen Sprechgeschwindigkeiten enthalten, zeigt, dass bei erhöhter Sprechgeschwindigkeit die relative Anzahl an Schwa-Elisionen im Schnitt um 24 \% höher liegt als im Lento. Diese Tatsache ist nicht auf einzelne SprecherInnen beschränkt, son-

\footnotetext{
${ }^{15}$ Siehe auch Hooper (1978: 190), der eine große individuelle Varianz bei der Schwa-Elision auch im amerikanischen Englisch feststellt.

${ }^{16} t$-Test: Kinder/Eltern: $t(8)=0,83$, n. s.; Eltern/Großeltern: $t(8)=0,83$, n. s.; Kinder/Großeltern: $t(8)=-0,23$, n. $\mathrm{s}$.
} 
dern der Unterschied liegt bei 10 von 15 SprecherInnen bei über $23 \%$ (bei einem Mininum von $5 \%$ und einem Maximum von $38 \%$ ). Bei starker sprecherindividueller Varianz trifft Hyp. 1 auf die Gesamtzahl der SprecherInnen zu, unabhängig von Alter oder Geschlecht. Zusätzlich kann diese mit durchschnittlich $64 \%$ in der unbewussten, flüssigen Redeweise (Allegro) und $40 \%$ in der bewussten, betonteren Redeweise (Lento) als ein im Lb. allgemein sehr häufiges Phänomen eingestuft werden.

\section{Der phonologische Kontext als Steuerungsfaktor der Schwa-Elision}

In der Beschreibung der Schwa-Elision unter Punkt 2 wurde die Bedeutung der phonologischen Umgebung des Vokals hervorgehoben. Im Folgenden wird untersucht, ob der SchwaVokal im Lb. in bestimmten Lautverbindungen häufiger elidiert wird als in anderen. Zeigt sich eine deutliche (nicht-) Elisionstendenz im Umfeld bestimmter Laute, erhärtet dies die Vermutung, dass dem Phänomen (auch) artikulatorische Gründe zugrunde liegen. Die zweite Hypothese, die geprüft wird, lautet:

- Hypothese 2: Bestimmte Lautverbindungen begünstigen die Schwa-Elision im Luxemburgischen.

Um festzustellen, in welcher Umgebung der Schwa-Vokal am stärksten der Elision unterliegt, wurden die im Experiment untersuchten Wörter sechs Untergruppen zugeordnet. ${ }^{17}$ Tabelle 5 stellt die Konsonantenverbindungen der jeweiligen Gruppen zusammen, zwischen denen Schwa in diesen Wörtern steht. Es steht dabei zumeist vor, gelegentlich nach oder zwischen Sonorant(en). Die erste Zahl in den Spalten „Anzahl“ bezeichnet die Menge an Wörtern im Lento (L), die zweite diejenige im Allegro (A). Für jede Untergruppe ist ein prototypisches Beispiel angeführt.

\begin{tabular}{|c|c|c|c|}
\hline Umgebung & $\begin{array}{l}\text { Anzahl } \\
\text { (L/A) }\end{array}$ & Umgebung & $\begin{array}{l}\text { Anzahl } \\
\text { (L/A) }\end{array}$ \\
\hline $\begin{array}{l}\text { (1) Plosiv }+ \text { o + Sonorant }- \\
\text { Elt(e)ren }\end{array}$ & $23 / 33$ & $\begin{array}{l}\text { (3) Liquid + }+ \text { + Obstruent }- \text { bëllegen }^{18} \\
\text {,billiger }\end{array}$ & $3 / 3$ \\
\hline (1a) Plosiv $+\partial+r$ & $11 / 20$ & (4) Nasal + ə + Liquid - Tromm(e)ler & $4 / 4$ \\
\hline (1b) Plosiv $+a+1$ & $10 / 11$ & (4a) Nasal $+2+r$ & $1 / 1$ \\
\hline (1c) Plosiv $+ə+$ Nasal & $2 / 2$ & (4b) $m,(n g)+\partial+1$ & $3 / 2(1)$ \\
\hline $\begin{array}{l}\text { (2) Frikativ }+ \text { o + Sonorant }- \\
\text { Konf(e)renz }\end{array}$ & $2 / 8$ & $\begin{array}{l}\text { (5) Liquid + ə + Nasal - Pill(e)men } \\
\text {,Kissen' }\end{array}$ & $1 / 1$ \\
\hline (2a) Frikativ $+\partial+r$ & $1 / 3$ & (6) Diphthong + o $+\mathrm{r}-\mathrm{Bau}(\mathrm{e}) \mathrm{ren}$ & $3 / 4$ \\
\hline (2b) Frikativ $+a+1$ & $0 / 3$ & & \\
\hline (2c) Frikativ $+2+n$ & $1 / 2$ & & \\
\hline
\end{tabular}

Tabelle 5: Konsonantische Umgebungen vor und nach Schwa im Lento/Allegro (= L/A) und Anzahl Testwörter pro Umgebung

\footnotetext{
17 Wörter mit elisionsfähigem Schwa in der Kombination Diphthong + ə + 1 und Diphthong + ə + Nasal sind sehr selten und häufig altertümlich (etwa Kréielchen ,kleiner Krug'). Sie wurden in der Studie daher nicht berücksichtigt.

$18<\mathrm{g}>$ wird in bëllegen frikativisch [3] ausgesprochen, siehe Gilles/Trouvain (2013).
}

ISSN 1615-3014 
Tabelle 6 führt für jede Untergruppe die Häufigkeiten der Elision (in Prozent) für die Gesamtheit der SprecherInnen im Lento/Allegro und in der Gesamtheit der Fälle auf. Zusätzlich sind die Unterschiede zwischen Allegro und Lento sowie zwischen den elidierten und nichtelidierten Fällen vermerkt.

\begin{tabular}{|c|c|c|c|c|c|c|c|c|c|c|c|c|c|}
\hline \multirow[b]{2}{*}{ Gruppe } & \multicolumn{3}{|c|}{$\begin{array}{l}\text { Allegro } \\
\text { (in \%) }\end{array}$} & \multicolumn{3}{|c|}{$\begin{array}{l}\text { Lento } \\
\text { (in \%) }\end{array}$} & \multicolumn{3}{|c|}{$\begin{array}{l}\text { Gesamt } \\
\text { (in \%) }\end{array}$} & \multicolumn{3}{|c|}{$\begin{array}{l}\text { Unterschied } \\
\text { Allegro/Lento } \\
\text { (in \%) }\end{array}$} & \multirow[t]{2}{*}{$\begin{array}{l}\text { Unterschied } \\
\text { elidiert/nicht- } \\
\text { elidiert } \\
(\text { in } \%) \\
\end{array}$} \\
\hline & $\overline{\mathbf{e}}$ & $\mathbf{k}$ & $\bar{u}$ & $\overline{\mathrm{e}}$ & $\mathbf{k}$ & $\bar{u}$ & $\overline{\mathbf{e}}$ & $\mathbf{k}$ & $\mathbf{u}$ & $\mathbf{e}$ & $\mathbf{k}$ & $\mathbf{u}$ & \\
\hline $\begin{array}{l}\text { (1) Plosiv + } 2 \\
+ \text { Sonorant }\end{array}$ & 73 & 19 & 8 & 37 & 53 & 10 & 58 & 33 & 9 & 36 & -34 & 2 & 25 \\
\hline $\begin{array}{l}\text { (2) Frikativ }+ \\
\partial+\text { Sonorant }\end{array}$ & 74 & 14 & 12 & 24 & 68 & 8 & 59 & 29 & 11 & 50 & -54 & -4 & 30 \\
\hline $\begin{array}{l}\text { (3) Liquid + } ə \\
+ \text { Obstruent }\end{array}$ & 20 & 78 & 1 & 33 & 67 & 0 & 27 & 72 & 1 & -13 & 11 & -2 & -45 \\
\hline $\begin{array}{l}\text { (4) Nasal + ə } \\
+ \text { Liquid }\end{array}$ & 47 & 24 & 29 & 48 & 33 & 20 & 47 & 28 & 24 & -1 & -9 & -8 & 19 \\
\hline $\begin{array}{l}\text { (5) Liquid + } ~ \\
+ \text { Nasal }\end{array}$ & 7 & 78 & 15 & 4 & 92 & 4 & 6 & 85 & 9 & 3 & -14 & -11 & -79 \\
\hline $\begin{array}{l}\text { (6) Diphthong } \\
+ə+r\end{array}$ & 33 & 25 & 42 & 59 & 13 & 28 & 46 & 19 & 35 & -26 & 12 & -14 & 27 \\
\hline
\end{tabular}

Tabelle 6: Elision (e), keine Elision (k) und unklare Fälle (u) von Schwa im Lento, Allegro und in der Gesamtheit (Allegro + Lento), daneben die Unterschiede zwischen Allegro und Lento sowie zwischen den elidierten und nicht-elidierten Fällen, gruppiert nach den einzelnen konsonantischen Umgebungen. Werte von mind. $50 \%$ stehen in Fettdruck

Die höchsten Elisionswerte im Allegro zeigen sich bei den Verbindungen Plosiv + a + Sonorant (73\%, etwa Elt(e)ren), und Frikativ + o + Sonorant (74\%, etwa Konf(e)renz). Die Gruppen 3 (bëll(e)gen) und 5 (Pill(e)men) hingegen zeigen mit jeweils um $78 \%$ hohe Werte bei den nicht-elidierten Formen. Im Vergleich zu den jeweiligen Entsprechungen im Lento reduzieren sich diese Werte um etwa die Hälfte bei Gruppe 1 (36\%), bei Gruppe 2 sogar um zwei Drittel (50\%). Die Verbindungen Plosiv $+a+$ Sonorant und Frikativ + a + Sonorant sind in der schnelleren Sprechgeschwindigkeit also mehr als die anderen Verbindungen von der Elision betroffen.

Gruppe 4 (Tromm(e)ler) befindet sich auf dem dritten Rang, obgleich der Unterschied zwischen elidierten und nicht-elidierten Formen sowohl im Lento als auch im Allegro verglichen mit den Gruppen 1 und 2 nicht sonderlich hoch ist. Bei ihr ist die hohe Zahl an unklaren Fällen jedoch prägnant: Sie macht im Schnitt ein Viertel aller Fälle aus. Die Schwa-Laute erscheinen bei Nasal + o + Liquid gehäuft entweder stark reduziert, oder aber die Umstellung der Artikulatoren lässt einen nicht weiter bestimmbaren Laut entstehen. In vielen Fällen ist es ohne maschinell gestützte Untersuchungen schwer herauszuhören, ob ein Schwa vorhanden ist oder nicht. 
Gruppe 3 und 5 zeigen die niedrigsten Elisionswerte. In beiden Gruppen steht vor dem Schwa ein Liquid. Obschon aufgrund der kleinen Zahl an Beispielen in diesen beiden Gruppen Vorsicht geboten ist, kann angenommen werden, dass Schwa nach Liquid stabiler ist als vor Liquid.

Bei Gruppe 6 (Bau(e)ren) springen insbesondere die mit durchschnittlich $35 \%$ höchsten unklaren Werte ins Auge. In der Tat konnte in vielen Fällen nicht eindeutig bestimmt werden, ob dem Diphthong ein weiterer (Schwa-)Vokal folgte oder nicht. Insgesamt ist die Elision häufiger als die nicht-Elision. Als einzige Gruppe zeigt sie auch im Lento Werte über $50 \%$. Schwa erscheint in Verbindung mit Diphthongen also selbst bei bewusster Aussprache sehr labil.

Eine stichhaltige Erklärung für diese Ergebnisse findet sich in der Sonorität der Konsonanten vor und nach Schwa. Hooper (1978) hat für das amerikanische Englisch nachgewiesen, dass Schwa zwischen zwei Konsonanten nur dann getilgt werden kann, wenn der Konsonant hinter Schwa sonorer ist als derjenige vor Schwa, etwa in $\operatorname{sep}(a)$ rate oder awf(u)lly. In diesen Fällen stellen die beiden Konsonanten nach Schwa-Ausfall universal präferierte CC-Onsetcluster ([pr] und [fl]) dar, mit einer zum Nukleus hin aufsteigenden Sonorität. Ein durch SchwaElision entstandenes Cluster, das der Sonoritätshierarchie nicht gerecht wird, blockiert hingegegen die Elision bzw. kommt nur sehr selten vor (etwa in ult(i)mate oder par(a)noic).

Die untersuchten Umgebungen in vorliegender Arbeit entsprechen genau den in Hooper (1978) beschriebenen Verhältnissen. So bilden die in Gruppen (1) und (2) aufgrund von Schwa-Elision freigewordenen Konsonanten ein neues, im Hinblick auf die Sonoritätshierarchie wohlgeformtes Anlautcluster (etwa El.te.ren > El.tren). Auch Gruppe 3 (Tromm(e)ler) wird, bei Analyse des Nasals als ambisilbisch, der Hierarchie gerecht: Trome.ler > Tromler. Umgebungen 3, 5 und 6, bei denen die Schwa umgebenden Konsonanten keine aufsteigende Sonorität aufweisen (Liquid + Obstruent, Liquid + Nasal bzw. Diphthong + Liquid), zeigen entsprechend die geringsten Elisionswerte.

Hyp. 2 trifft also zu: Bestimmte Lautverbindungen begünstigen die Elision des SchwaVokals. Die Schwa-Elision im Lb. ist vor und nach Sonoranten jedoch in allen im Experiment auftretenden Verbindungen möglich. Sie tritt allerdings häufiger in den Verbindungen Plosiv/Frikativ $+a+$ Sonorant auf als bei initialem Liquid, was u. a. mit universalen Silbenstrukturbeschränkungen (Sonoritäts-Hierarchie) in Verbindung gesetzt werden kann. In der Umgebung von Nasalen zeigt sich eine erhöhte Frequenz von unklaren Fällen. In der Umgebung von Diphthongen ist eine eindeutige Zuweisung ebenfalls erschwert. ${ }^{19}$ Diese Erkenntnisse decken sich mit denjenigen im Dt. (Kontext a), zeigen aber Unterschiede zwischen dem Lb. und dem Ndl., in dem kategorisch nur Schwa zwischen Obstruent + a + Liquid betroffen ist (siehe Punkt 2).

\footnotetext{
${ }^{19}$ Unter Punkt 4.3.2 wurden große sprecherindividuelle Unterschiede bei den unklaren Fällen festgestellt, was die Aussagekraft dieser Zahlen etwas reduziert.
}

ISSN 1615-3014 


\section{Die Rolle des Schwa-Vokals in der luxemburgischen Wortprosodie}

Wie die vorherigen Punkte gezeigt haben, spielen realisationsphonetische und artikulatorische Aspekte bei der Erklärung der Schwa-Elision eine Rolle. Der Bereich der Wortprosodie ist jedoch ebenfalls betroffen, da die Schwa-Elision einen direkten Einfluss auf die Einheit des prosodischen Fußes hat. Durch den Wegfall einer Silbe wird ein Daktylus zu einem Trochäus: Eemeren (3-silb.) > Eemren (2-silb.) ,Eimer' (P1.). ${ }^{20}$

Aus Mangel an lb. Forschungsliteratur wird erneut der Weg über die dt. Forschung gewählt. Im Vergleich mit der Arbeit von Peter Eisenberg zur „Prosodik deutscher Wörter“ (Eisenberg: 1991) wird im Folgenden die prosodische Grundstruktur der lb. Substantive, Adjektive und Verben dargestellt und anschließend mit der Schwa-Elision in Beziehung gesetzt. ${ }^{21}$

Eisenberg folgend wähle ich für meine Ausführungen stets die längstmögliche Form der Wörter. Es handelt sich also nicht immer um die Grundform, sondern oftmals um Wortformen mit Flexionsaffixen. ${ }^{22}$ Zudem beschränke ich mich bei der Beschreibung wie Eisenberg vor allem auf den von ihm definierten „Kernbereich“ sowie Simplizia. ${ }^{23}$ Um eine möglichst fruchtbare Vergleichbarkeit mit der dt. Studie zu gewährleisten, werden für die Beispielwörter bei Eisenberg lb. Entsprechnungen gewählt, die diesen möglichst ähnlich sind. ${ }^{24}$

\subsection{Die prosodische Struktur der flektierten Einheiten I: Substantive (Singular)}

Eisenberg (1991) beschreibt zwei „Hauptklassen“ der zweisilbigen Substantive: (1) diejenigen mit Schwasilbe (Hütte, Hammer) und (2) diejenigen mit zwei betonbaren Silben (Uhu, Echo). Da die erste Gruppe die „überwältigende Mehrheit der Substantive im Kernbereich“ enthält, spricht Eisenberg (1991: 47) von der „kanonischen Struktur des Substantivs im Deutschen". Entsprechend der Unbetonbarkeit der zweiten Silbe liegt die Betonung immer auf der ersten Silbe, es handelt sich also stets um einen Trochäus. Als Auftrittskontexte des SchwaVokals nennt er (1a) Schwa als Teil des Stamms und in offener Endsilbe (Hase, Funke, Straße, Hütte, Auge), (1b) Schwa als Teil des Stamms und vor einfachem Auslaut mit Sonorant (Eimer, Esel, Leben, Atem) und (1c) Schwa (fakultativ) im Flexiv einsilbiger Substantive

\footnotetext{
20 Der prosodische Fuß besteht nach Hall (2000: 277) aus „,einer betonten Silbe und den unbetonten Silben bis zum nächsten Fuß“, d. h. bis zur nächsten betonten Silbe. Der Trochäus stellt in der Struktur ,,betonte Silbe + unbetonte Silbe“ den ,in den Sprachen der Welt [...] am wenigsten markierte[n] Fuß“ (Hall ibd.) dar: Bsp. 'Ie.sel ,Esel`. Laut Gilles (2010) ist er neben dem Dt. auch im Lb. vorherrschend. Der Daktylus besteht aus drei Silben. Einer betonten Silbe folgen zwei unbetonte Silben, etwa in 'Dak.ty.lus.

${ }^{21}$ Weitere Untersuchungen zur deutschen prosodischen Struktur finden sich etwa bei bei Féry (1991) und Wiese (1996). Die Fußstruktur des Niederländischen ist etwa bei Booij (1995) beschrieben. Aufgrund des klaren Aufbaus und der damit verbundenen einfachen Vergleichbarkeit zwischen dem Deutschen und dem Luxemburgischen wird sich in diesem Artikel auf Eisenberg beschränkt.

22 „Ist von einer syllabischen Struktur von Wörtern (Flexionsparadigmen) die Rede, so ist damit eine syllabische Struktur einer Form des Paradigmas mit maximaler Silbenzahl gemeint“ (Eisenberg 1991: 46). Eisenberg hat für das Dt. zudem die „Explizitlautung“ (ohne silbische Konsonanten und ohne andere Reduktionsvokale als [ə]) gewählt. In diesen Formen ist [ə] auch stets nicht elidiert.

23 Der Kernbereich beinhaltet laut Eisenberg die „nativen“, nicht-suffigierten Wörter mit einfachem, einsilbigem Stamm. Die empirische Untersuchung der vorliegenden Arbeit beinhaltet auch einige (integrierte) Fremdwörter (diese haben jedoch die gleiche prosodische Struktur, siehe Anhang a). Zudem wurden einige suffigierte Wörter (etwa adeleg , adlig') eingebaut. Wie die Ergebnisse zeigen (siehe weiter unten), verhält sich die Schwa-Elision hier nicht anders als bei den Wörtern des Kernbereichs.

${ }^{24}$ Diese stellen zum Teil erst kürzlich aus dem Dt. entlehnte Wörter dar und werden von einigen älteren SprecherInnen möglicherweise nicht häufig genutzt. Sie sind jedoch auch für diese vollkommen verständlich.
}

ISSN 1615-3014 
(Bsp. Bär-(e)n, Ball-(e)s und Haus-(e)s). ${ }^{25}$ Alle Substantive der Gruppen (1) und (2) entsprechen also in ihrer „maximalen Silbenstruktur” im gesamten Flexionsparadigma dem kanonischen Trochäus.

Aufgrund des Ausfalls des Schwa-Vokals in der ungedeckten Endsilbe (a-Apokope, siehe Conrad 2010; Szczepaniak 2010) fällt im Lb. Gruppe (1a) (Typ Hase, lb. Hues) aus. Neben dem Trochäus erscheint daher gehäuft der Einsilbler. Die Substantive der Untergruppe (1b) verhalten sich in der Regel wie die dt. Entsprechungen (etwa Eemer ,Eimer', Iesel ,Esel', Liewen ,Leben', Otem ,Atem'). Da das 1b. Substantiv bis auf wenige Ausnahmefälle nicht flektiert wird (Schanen/Zimmer 2006a), fällt auch Gruppe (1c) aus: Alle in der Grundform trochäischen Substantive im Singular behalten diese prosodische Struktur im gesamten Flexionsparadigma. ${ }^{26}$

\subsection{Die prosodische Struktur der flektierten Einheiten II: Substantive (Plural)}

Auch für den Plural postuliert Eisenberg (1991) für das Dt. den kanonischen Trochäus. Die zweisilbigen Wörter der Gruppen (1a) und (1b) erhalten entweder kein Flexiv (der Eimer die Eimer) oder ein konsonantisches Flexiv (der Hase - die Hasen): Der Trochäus bleibt in jedem Fall erhalten. Die Substantive der Gruppe (1c), die in ihrer Grundform einsilbig sind, erhalten jeweils ein Schwa-Flexiv ([ə], [ən] oder []]), etwa der Ball-die Bälle, der Bär - die Bären, das Haus - die Häuser. Auch sie werden somit trochäisch.

Im Lb. verhält es sich anders. Bis auf die Formen, die einen Umlaut zeigen (etwa Apel - Äppel ,Apfel - Äpfel‘, Wollef - Wëllef ,Wolf - Wölfe') und einige Wörter, die einsilbig bleiben (etwa Stull - Still ,Stuhl - Stühle', Numm - Nimm ,Name - Namen', Schwäin - Schwäin ,Schwein - Schweine') erhalten alle Substantive ein Flexiv mit Schwa, entweder -en oder -er: Eemer - Eemeren ,Eimer - Eimer', Iesel - Ieselen ,Esel - Esel' (1b), Bier - Bieren ,Bär Bären' (1c). ${ }^{27}$ Einsilbige Wörter werden so in der Regel trochäisch. Mit Ausnahme der umgelauteten Formen werden aus den zweisilbigen Substantiven dreisilbige Wörter - aus Trochäen werden im Plural häufig Daktylen.28

Für Gruppe (2) zeigt sich ein vergleichbares Bild: Verhalten sich Wörter wie Uhu oder Echo im Dt. wie die Wörter der Gruppe (1b), indem sie in allen Fällen trochäisch bleiben (Plural: Uhus, Echos), erscheinen sie im Lb. nur im Singular als Trochäus. Im Plural wird durch das Anhängen des Flexivs -en erneut ein Daktylus gebildet (Uhuen, Echoen). ${ }^{29}$

\footnotetext{
${ }^{25}$ Die Bezeichnungen (1a)-(1c) stammen vom Autor dieses Artikels.

${ }^{26}$ Erwähnenswert sind zudem die wenigen Wörter mit eingeschobenem Schwa-Vokal (Schwa-Einschub, siehe Krier 2008; Conrad 2010; Szczepaniak 2010; Gilles 2014), die anders als im Dt. ebenfalls zweisilbig sind und im gesamten Paradigma zweisilbig bleiben (etwa 'Wollef, Wolf', 'Hëllef ,Hilfe', 'Mëllech ,Milch'); siehe zu diesem Phänomen im Nied. Booij (1995: 127-128).

27 Zur Pluralbildung im Lb. allgemein siehe etwa Schanen/Zimmer (2006a).

${ }^{28}$ Eine Ausnahme stellen die Substantive dar, die auf -en auslauten: Diese bleiben zweisilbig/trochäisch: Uewen - Iewen ,Ofen - Öfen'; Liewen - Liewen ,Leben - Leben`. Einige wenige Wörter zeigen Umlaut und Endung, etwa Haus - Haiser ,Haus - Häuser' (für diesen Hinweis danke ich Cristian Kollmann).

${ }^{29}$ Schanen/Zimmer (2006a: 34) nennt auch ein paar ,isolierte“ Fälle, in denen nur die trochäische oder aber eine trochäische und eine daktylische Variante fakultativ zur Verfügung stehen, etwa Bopa - Bopen ,Opa - Opas', aber Villa - Villen/Villaen ,Villa - Villas‘. Auch die Mehrzahl der Fremdwörter erhält als Pluralflexiv -en (ibd.: $33)$.
}

ISSN 1615-3014 
Die jeweilige Prosodik der Substantive der „Kerngruppe“ im Dt. und im Lb. wird in Tabelle 7 gegenübergestellt:

\begin{tabular}{|c|c|c|c|c|}
\hline Gruppe: & $\begin{array}{c}\text { (1a) } \\
\text { Hase/Hues }\end{array}$ & $\begin{array}{c}\text { (1b) } \\
\text { Eimer/Eemer }\end{array}$ & $\begin{array}{c}\text { (1c) } \\
\text { Bär/Bier }\end{array}$ & $\begin{array}{c}(2) \\
\text { Echo/Echo }\end{array}$ \\
\hline \multicolumn{5}{|l|}{ Singular } \\
\hline Deutsch & Trochäus & Trochäus & Trochäus & Trochäus \\
\hline Luxemburgisch & Einsilbig & Trochäus & Einsilbig & Trochäus \\
\hline \multicolumn{5}{|l|}{ Plural } \\
\hline Deutsch & Trochäus & Trochäus & Trochäus & Trochäus \\
\hline Luxemburgisch & $\begin{array}{l}\text { Trochäus } \\
\text { /Einsilbig }\end{array}$ & $\begin{array}{l}\text { Daktylus } \\
\text { (/Trochäus) }\end{array}$ & $\begin{array}{l}\text { Trochäus } \\
\text { /Einsilbig }\end{array}$ & Daktylus \\
\hline
\end{tabular}

Tabelle 7: Maximale Silbenstruktur im Sg./Pl. der Substantive der Gruppen (1a)-(2) im Dt. und Lb. mit prototypischen Beispielen (Dt./Lb.). Die Form in Klammern ist markiert. Nicht-trochäische Fußtypen stehen zur besseren Abhebung kursiv

Im Singular beruhen die geringen Unterschiede (mehr einsilbige Formen im Lb.) auf morphologischen Faktoren, etwa der ə-Apokope und der Flexion. Im Plural erscheint im Lb. in der Mehrheit der Fälle im Vergleich zum Dt. eine zusätzliche Silbe. Diese stellt somit einen Pluralmarker dar, der im Dt. in dieser Form nicht (mehr) auftritt. Für den Bereich der Wortprosodie der lb. Substantive im Plural begrenzt der (morphologische) Pluralmarker also eine (prosodisch) einheitliche Fußbildung. ${ }^{30}$ Demgegenüber erlangt (oder bewahrt) das dt. Substantiv in allen Formen die unmarkierte trochäische Struktur. Im Dt. dominiert hier also die Prosodie. Diese Aussagen werden unter 6.6.5 erneut aufgegriffen.

\subsection{Die prosodische Struktur der flektierten Einheiten III: Adjektive}

Eisenberg zufolge ist

jede flektierte Form eines Adjektives [...] mindestens zweisilbig und hat dann die Betonungsstruktur $\left\{{ }^{-},-\right\}$[= Trochäus, F. C.]. Tritt zu einer zweisilbigen Form ein weiteres silbisches Suffix, so fällt stammbildendes Schwa immer aus (müde - müdes) und ebenso Schwa vor [1] (edel - edles). In allen anderen Fällen ist der Ausfall von Schwa fakultativ (offen - offenes offnes, munter - munteres - muntres). ${ }^{31}$

(1991: 51)

$\mathrm{Zu}$ den stammbildenden Endungen mit Schwa bei dt. Adjektiven zählt er -er (heiter, munter), -el (eitel, edel), -en (offen, trocken) und -e (müde, rege). Das dt. flektierte Adjektiv ist also nie einsilbig. Neben dem (in einigen Fällen fakultativen) Trochäus tritt häufig auch der Daktylus auf. Somit stehen bei den dt. Adjektiven anders als bei den Substantiven zwei Fußstrukturen zur Verfügung.

Auch in diesem Bereich unterscheidet sich das Lb. vom Dt. Erneut sind die Gründe zunächst in der Morphologie zu suchen: Aufgrund der ə-Apokope sind viele im Dt. zweisilbige Formen im Lb. einsilbig (etwa midd, müde'). Daneben unterscheidet das Lb. nur selten zwischen star-

\footnotetext{
${ }^{30}$ Vgl. Gilles (2014: 289): „[...] the final syllable carries important morphological information (e. g. number, case or person), which in Luxembourgish seems to be protected from reduction."

${ }^{31}$ Hervorhebungen: F. C.
} 
ker und schwacher Adjektiv-Flexion und nur die Dativ-Endungen (in allen Genera und im Plural) sowie alle maskulinen Formen zeigen silbische Suffixe (-en, -er oder seltener -em) (ausführlich etwa Schanen/Zimmer 2006a). Dadurch bleiben eine Reihe von Adjektiven auch flektiert einsilbig (midd - ech gesinn eng midd Kaz ,müde - ich sehe eine müde Katze').

Daneben gibt es aber auch Formen, die sich z. T. wie die dt. Entsprechungen verhalten. So sind die stammbildenden Endungen mit Schwa im Lb. ebenfalls -er (heiter ,heiter', monter ,munter'), -el (eitel ,eitel', edel ,edel') und -en (oppen ,offen“, dréchen ,trocken'). Aus diesen trochäischen Formen werden durch Anhängen eines silbischen Suffixes Daktylen, anders als im Dt. auch bei -el (en eitele Mann ,ein eitler Mann'). Bis auf nähere Prüfung weiter unten wird im Lb. die maximale, daktylische Form als unmarkiert eingestuft, neben einer optionalen, trochäischen Form (Schanen/Zimmer 2006b: 51-52).

Tabelle 8 stellt die prosodische Struktur der dt. und lb. Adjektive gegenüber.

\begin{tabular}{|l|l|l|l|l|l|}
\hline Grundform & einsilbig & $\begin{array}{l}\text { zweisilbig } \\
\text { auf }-\boldsymbol{e}\end{array}$ & $\begin{array}{l}\text { zweisilbig } \\
\text { auf -el } \\
\text { eitel/eitel }\end{array}$ & $\begin{array}{l}\text { zweisilbig } \\
\text { auf -en }\end{array}$ & $\begin{array}{l}\text { zweisilbig auf } \\
-\boldsymbol{e r}\end{array}$ \\
\hline $\begin{array}{l}\text { Beispiel } \\
\text { Flektierte Formen } \\
\text { (silbisches Flexiv) }\end{array}$ & müde/midd & munter/monter \\
\hline Deutsch & Trochäus & Trochäus & Trochäus & $\begin{array}{l}\text { Daktylus } \\
\text { (/Trochäus) }\end{array}$ & $\begin{array}{l}\text { Daktylus } \\
\text { (/Trochäus) }\end{array}$ \\
\hline $\begin{array}{l}\text { Luxembur- } \\
\text { gisch }\end{array}$ & $\begin{array}{l}\text { Einsilbig/ } \\
\text { Trochäus }\end{array}$ & & $\begin{array}{l}\text { Daktylus } \\
\text { (/Trochäus) }\end{array}$ & $\begin{array}{l}\text { Daktylus } \\
\text { (/Trochäus) }\end{array}$ & $\begin{array}{l}\text { Daktylus } \\
\text { (/Trochäus) }\end{array}$ \\
\hline
\end{tabular}

Tabelle 8: Maximale Silbenstruktur der Adjektive im Dt. und Lb. mit prototypischen Beispielen (Dt./Lb.). Die Formen in Klammern sind markiert. Nicht-trochäische Fußtypen stehen zur besseren Abhebung kursiv

Erneut zeigt das Lb. somit eine größere Varianz als das (Standard)Dt.: Neben dem Trochäus finden sich vermehrt einsilbige und daktylische Formen.

\subsection{Die prosodische Struktur der Verben}

Laut Eisenberg ist die prosodische Struktur der Verben im Dt. „noch weniger einheitlich und strikt als bei den Adjektiven“ (1991: 53). Er beschränkt sich daher auf den Infinitiv, das Präsens sowie das Imperfekt von schwachen Verben und unterteilt diese in drei Gruppen: (1) Verben mit einsilbigen Stämmen (legen, beten), (2) Verben mit Schwasilben (angeln, nörgeln, scheitern, plappern) und (3) Verben mit nichtsilbischem Nasal im Stammauslaut (atmen, widmen, regnen, ebnen). ${ }^{32}$ Erneut wird für das Lb. der gleiche Weg eingeschlagen, jedoch wird das im Lb. seltene Imperfekt ausgeklammert. ${ }^{33}$

Neben einigen einsilbigen Formen im Präsens (du legst, er führt) sowie wenigen (fakultativ) daktylischen Formen (ich seg(e)le, ich scheit(e)re) zeigen die dt. Infinitive und Präsensformen durchgängig den „kanonischen“ Trochäus.

\footnotetext{
32 Die Benennungen (1)-(3) stammen nicht von Eisenberg (1991), sondern vom Autor dieses Artikels.

33 Der Imperfekt ist im Lb. in den allermeisten Fällen dem Perfekt gewichen (siehe etwa Braun et al. 2005: 2021).
} 
Das Lb. gestaltet sich erneut etwas anders. Grund hierfür ist insbesondere die „Synkoperesistenz" des Schwa im Infinitiv-Suffix und in den Verbalendungen, das immer realisiert wird (Gilles 1998; Nübling 2005; Krier 2008). Gruppe (2) muss im Lb. daher erweitert werden zu „Verben mit Schwa-Silben im Stamm“. Da im Lb. silbische Konsonanten gemieden werden, muss auch Gruppe (3) terminologisch angepasst werden. Ausreichend ist hier die Bezeichnung „Nasal im Stammauslaut““.

Die Verben der Gruppe (1) und (2) (etwa kucken ,schauen, gucken', bieden ,beten' (1); ootmen ,atmen', widmen ,widmen', reenen ,regnen', zeechnen, zeichnen' (2)) verhalten sich im Infinitiv wie die dt. Entsprechungen und bilden einen Trochäus. Gruppe (3) verhält sich aber anders: Aufgrund der konsequenten Realisierung der vollen Endung [ən] entspricht dem dt. Trochäus im Lb. ein Daktylus: aangelen , angeln', nörgelen ,nörgeln', scheiteren ,scheitern'.

Auch in den Präsensformen gibt es teils große Unterschiede. ${ }^{34}$ Tabelle 9 stellt jeweils zwei Beispiele der Gruppen (1)-(3) gegenüber.

\begin{tabular}{|l|l|l|l|l|l|l|}
\hline Gruppe & \multicolumn{3}{l|}{$\begin{array}{l}\text { (1) einsilbiger } \\
\text { Stamm }\end{array}$} & \multicolumn{2}{l|}{$\begin{array}{l}\text { (2) Schwa-Silbe im } \\
\text { Stamm }\end{array}$} & \multicolumn{2}{l|}{$\begin{array}{l}\text { (3) Nasal im Stammaus- } \\
\text { laut }\end{array}$} \\
\hline Infinitiv & kucken & bieden & scheiteren & seegelen & zeechnen & ootmen ${ }^{35}$ \\
\hline 1. Pers. Sg. & kucken & bieden & scheiteren & seegelen & zeechnen & ootmen \\
\hline 2. Pers. Sg. & kucks & biets & scheiters & seegels & zeechens & otems \\
\hline 3. Pers. Sg. & kuckt & biet & scheitert & seegelt & zeechent & otemt \\
\hline 1. Pers. Pl. & kucken & bieden & scheiteren & seegelen & zeechnen & ootmen \\
\hline 2. Pers. Pl. & kuckt & biet & scheitert & seegelt & zeechent & otemt \\
\hline 3. Pers. Pl. & kucken & bieden & scheiteren & seegelen & zeechnen & ootmen \\
\hline
\end{tabular}

Tabelle 9: Flexionsparadigma lb. Verben der Gruppen (1)-(3) im Präsens (für die Untersuchung relevante Unterschiede zum Dt. stehen in Fettdruck $)^{36}$

Bei den Verben der Gruppe (2) findet sich aufgrund der gleichen Endung für 1. Pers. Sg./Pl. und 3. Pers. Pl. und der nicht-reduzierten Aussprache von -en in drei Formen des Paradigmas Dreisilbigkeit (gegenüber nur einer dreisilbigen Form im Dt.). Daneben gibt es mehr einsilbige Formen in den Verben der Gruppe (1) (du biets, hien/hatt biet, dir biet, du betest, er/sie/es betet, ihr betet'). Wie bei den Substantiven und Adjektiven erhält der im Dt. kanonische Trochäus im Lb. Konkurrenz von Daktylen und Einsilblern.

Tabelle 10 stellt die Prosodik der (schwachen) dt. und lb. Verben vergleichend gegenüber.

\footnotetext{
${ }^{34}$ Ausführlich zur Konjugation der lb. Verben siehe etwa Braun et al. (2005) und Schanen/Zimmer (2005)

35 Für ootmen , atmen' existiert die alternative Form otemen, die in den Personen 1. Sg., 1. Pl. und 3 Pl. entsprechend einen Daktylus aufzeigt.

36 Übersetzung: siehe Fließtext weiter oben.
} 


\begin{tabular}{|l|l|l|l|}
\hline Gruppe & $\begin{array}{l}\text { einsilbiger } \\
\text { Stamm (1) } \\
\text { Beispiel }\end{array}$ & $\begin{array}{l}\text { Schwa-Silbe (im } \\
\text { Stamm) (2) } \\
\text { segeln/seegelen }\end{array}$ & $\begin{array}{l}\text { (nicht-silb.) Nasal im } \\
\text { Stammauslaut (3) }\end{array}$ \\
\hline zeichnen/zeechnen \\
\hline Deutsch & Trochäus & Trochäus & Trochäus \\
\hline Luxemburgisch & Trochäus & $\begin{array}{l}\text { Daktylus } \\
\text { (/Trochäus) }\end{array}$ & $\begin{array}{l}\text { Trochäus } \\
\text { (/Daktylus)(selten) }\end{array}$ \\
\hline Ind. Präsens & Trochäus & $\begin{array}{l}\text { Trochäus } \\
\text { (/Daktylus) }\end{array}$ & Trochäus \\
\hline Deutsch & /Einsilbig & $\begin{array}{l}\text { Daktylus } \\
\text { (/Trochäus) }\end{array}$ & Trochäus \\
\hline Luxemburgisch & $\begin{array}{l}\text { Trochäus } \\
\text { Einsilbig }\end{array}$ &
\end{tabular}

Tabelle 10: Maximale Silbenstruktur der (schwachen) Verben der Gruppen (1)-(3) im Infinitiv und Indikativ Präsens im Dt. und Lb. mit prototypischen Beispielen (Dt./Lb.). Die Formen in Klammern sind markiert. Nicht-trochäische Fußtypen stehen zur besseren Abhebung kursiv

\subsection{Zusammenfassung}

In Anlehnung an die „Prinzipien der Prosodik deutscher Wörter“ von Peter Eisenberg (1991) wurde die Wortprosodie der Substantive, Adjektive und Verben im „Kernbereich“ im Dt. und Lb. gegenübergestellt. Hierbei spielen die beiden Fußtypen Trochäus und Daktylus die zentrale Rolle.

Eisenberg hat gezeigt, dass im Dt. der Trochäus in den untersuchten Wortformen vorherrschend ist. Dieser gilt daher als „kanonische“ Fußstruktur des Dt. Einsilbige Formen sowie Daktylen erscheinen nur vereinzelt.

Auch im Lb. tritt der Trochäus sehr häufig auf. Allerdings erhält er Konkurrenz von einsilbigen und v. a. daktylischen Formen. Gründe hierfür sind unter anderem die ə-Apokope sowie weitere morphologische Erscheinungen (kaum Flexion der Substantive, nur wenige silbische Flexive bei den Adjektiven, häufiges silbisches Pluralsuffix). Daneben spielen phonologische Elemente eine Rolle, etwa die volle, nicht-reduzierte Artikulation der en-Endung.

In allen untersuchten Wortklassen finden sich im Lb. jedoch zahlreiche Wörter, die ein elisionsfähiges Schwa nach den unter Punkt 2 beschriebenen Aspekten (Schwa-Silbe nach betonter Silbe, sonorantische Umgebung) aufzeigen. Es handelt sich hierbei um Substantive der Gruppe (1b) im Plural (Eem(e)ren ,Eimer'), daktylische Adjektivformen (opp(e)nen ,offener') sowie daktylische Verbformen im Infinitiv und im Präsens der Gruppen (2) und (seltener) (3) (seeg(e)len ,segeln', ootmen ,atmen'). Einige solcher Wörter wurden im Experiment auf die Häufigkeit ihrer Schwa-Elision untersucht. Eine hohe Anzahl an Elisionen lässt die daktylischen Formen zugunsten der trochäischen im gesprochenen Lb. wieder zurücktreten. Die Schwa-Elision könnte entsprechend prosodisch motiviert sein: Um den kanonischen Trochäus wortartübergreifend zu erlangen, wird der in der Schrift stets präsente elisionsanfällige Schwa-Vokal im gesprochenen Lb. getilgt. Die Hypothese, die im nächsten Punkt überprüft wird, lautet dementsprechend: 
- Hypothese 3: Die Schwa-Elision ist prosodisch gesteuert und dient der Erlangung einer kanonischen trochäischen Fußstruktur.

\subsection{Ergebnisse der empirischen Untersuchung}

Im Folgenden werden für die Gruppen Substantive Singular, Substantive Plural, Adjektive (flektiert) und Verben im Infinitiv die Realisierungen der untersuchten Wörter im Allegro aufgezählt (in \%). Da es primär um die prosodische Form der Wörter im gesprochenen Lb. geht, wird die Lento-Form, sofern das Wort auch auf diese Weise auftrat, nur zu Vergleichszwecken aufgezählt (die Gesamtzahl, also Allegro + Lento, befindet sich in diesem Fall in Klammern hinter der Prozentzahl der Allegro-Form). Die Zahl in Klammern in der ersten Spalte zeigt die Silbenzahl der betroffenen Wörter (neben Daktylen finden sich ein paar mehrsilbige Wörter). Liegt der Wortakzent nicht auf der ersten Silbe, ist er im Wort mit einem Apostroph angegeben. Die Silbenzahl wird in dem Fall ab der betonten Silbe gezählt - es zählt hier also der Fuß, nicht das Wort. Werte von mind. 50 \% stehen in Fettdruck.

\subsubsection{Ergebnisse Substantive: Singular}

\begin{tabular}{|c|c|c|c|}
\hline Wort & Trochäus (in \%) & Daktylus (in \%) & unklar (in \%) \\
\hline Trommeler (3) & $59(53)$ & $7(10)$ & $34(37)$ \\
\hline Éisterräich (3) & $93(80)$ & $0(8)$ & $7(12)$ \\
\hline Prabbeli (3) & $83(54)$ & $7(16)$ & $10(30)$ \\
\hline Schokela (3) & 57 & 23 & 20 \\
\hline Braue'rei (3) & 21 & 46 & 32 \\
\hline Seegeler (3) & 80 & 7 & 13 \\
\hline Aangeler (3) 37 & 27 & 19 & 54 \\
\hline Gesamt: & $60 \%(62 \%)$ & $16 \%(11 \%)$ & $24 \%(26 \%)$ \\
\hline
\end{tabular}

Tabelle 11: Prosodische Realisierung der daktylischen Formen von Substantiven im Singular (Gruppe (1a), in \%) im Allegro. Gesamtzahl (Allegro + Lento) in Klammern, falls zutreffend

Gruppe (1a) zeigt in 60 \% der Fälle Reduktion von einem Daktylus zu einem Trochäus (im Allegro). In $16 \%$ der Fälle unterbleibt die Reduktion, rund ein Viertel aller Fälle (24 \%) ist unklar.

Bei den Wörtern, die in über 50 \% der Fälle Trochäen zeigen, ist der Konsonant links von Schwa sonorer als der Konsonant rechts von Schwa. Die zweite, nicht betonte Silbe des Wortes, die aus der Resilbifizierung der freigewordenen Konsonanten hervorgeht, entspricht somit der Sonoritätshierarchie (cf. hierzu Punkt 5). Zwei Wörter verhalten sich jedoch anders: Bei Brauerei dürfte sowohl die nicht eingehaltene Sonoritätshierarchie (der Glide im Diphthong ist sonorer als der Liquid nach Schwa) als auch die Endbetonung eine Rolle bei der hohen Zahl an Daktylen spielen, während bei Aangeler aus artikulatorischen Gründen (Verbindung zwischen velarem Nasal und Liquid) besonders häufig nicht festgestellt werden konnte, ob ein Schwa vorhanden war oder nicht.

37 Trommeler ,Trommler', Éisterräich ,Österreich‘, Prabbeli ,Regenschirm‘, Schokela ,Schokolade‘, Brauerei ,Brauerei', Seegeler ,Segler', Aangeler ,Angler'. 
Die Werte bei der Gesamtzahl der Fälle entsprechen im Groben denen der Allegro-Werte alleine. Somit treten bei den Substantiven im Singular bei unbewusst artikulierter Sprache etwa gleich viele Trochäen auf als bei einer bewusst artikulierten Sprache. Das Lb. zeigt also für einen Großteil der hier behandelten Substantive im Singular eine starke Tendenz, Trochäen zu bilden, obschon Daktylen möglich wären.

\subsubsection{Ergebnisse Substantive: Plural}

Die Wörter der Gruppe (1b) zeigen eine ähnliche Tendenz wie die Wörter in (1a) (Tabelle 12): In etwas mehr als der Hälfte der Fälle (57\%) wird ein Daktylus zu einem Trochäus reduziert (in drei Fällen ein 4-Silbler zu einem Daktylus), gegenüber $28 \%$, in denen der Daktylus erhalten bleibt. Die unklaren Fälle liegen mit $14 \%$ um $10 \%$ niedriger als bei den Substantiven im Singular. ${ }^{38}$ Erneut wird die Trochäus-Struktur bevorzugt. ${ }^{39}$

\begin{tabular}{|c|c|c|c|}
\hline Wort & reduziert (in \%) & nicht-reduziert (in \%) & unklar (in \%) \\
\hline Baueren (3) & $80(58)$ & $0(17)$ & $20(25)$ \\
\hline Mi'nisteren (3) & $57(29)$ & $36(68)$ & $7(4)$ \\
\hline Eemeren (3) & $11(7)$ & $74(84)$ & $15(9)$ \\
\hline Duechteren (3) & $56(28)$ & $37(69)$ & $7(4)$ \\
\hline Omeletten (4) & 87 (77) & $0(5)$ & $13(18)$ \\
\hline Pillemen (3) & $7(6)$ & $78(85)$ & $15(9)$ \\
\hline Gromperen (3) & 77 & 20 & 3 \\
\hline Ieselen (3) & 48 & 34 & 17 \\
\hline Seegelen (3) & 78 & 4 & 19 \\
\hline Restauranten (4) & 48 & 52 & 0 \\
\hline Ouschteren (3) & 90 & 3 & 7 \\
\hline Aueren $(3)^{40}$ & 47 & 3 & 50 \\
\hline Gesamt & $57 \%(34 \%)$ & $28 \%(55 \%)$ & $14 \%(12 \%)$ \\
\hline
\end{tabular}

Tabelle 12: Prosodische Realisierung der daktylischen/mehrsilbigen Formen von Substantiven im Plural (Gruppe (1b), in \%) im Allegro. Gesamtzahl (Allegro + Lento) in Klammern, falls zutreffend

\footnotetext{
${ }^{38}$ Hier spielen artikulatorische Gründe eine Rolle, siehe Punkt 5. Im Plural sind schlicht weniger Wörter vertreten, die unklar artikuliert wurden.

${ }^{39}$ Bei der Hinzuziehung der Lento-Formen zeigt sich, dass sich die Werte umkehren: Im Lento bleiben also wesentlich mehr Fälle in ihrer daktylischen Form. Für die Substantive im Plural in der schnelleren Rede heißt dies, dass Allegro, wie bereits unter Punkt 4 festgestellt, die Schwa-Elision und damit die Schaffung von Trochäen begünstigt, wohingegen im Lento die vollen Formen dominieren.

40 Baueren ,Bauern', Min 'isteren ,Minister', Eemeren ,Eimer', Duechteren ,Töchter' (alternative, ältere Form: Diechter), Omeletten ,Omletts‘, Pillemen ,Kissen“, Gromperen ,Kartoffeln“, Ieselen ,Esel‘, Seegelen ,Segel', Restauranten ,Restaurants', Ouschteren ,Ostern', Prabbelien ,Regenschirme', Aueren ,Uhren“.
} 


\subsubsection{Ergebnisse Adjektive (flektiert)}

Die Werte für Gruppe (2a) fallen verhältnismäßig hoch aus (Tabelle 13): In fast drei Viertel aller Fälle (71 \%) wird im Allegro eine potentiell daktylische Form trochäisch realisiert, bzw. eine 4-silbige Form gekürzt. Ein Viertel der Fälle bewahrt die daktylische Struktur, bei nur $5 \%$ unklaren Formen.

Die Werte verändern sich bei Hinzuziehung der Lento-Formen erneut leicht um etwa $10 \%$, so dass die für Gruppe (1a) getroffene Schlussfolgerung auch für Gruppe (2a) zutrifft: Das Lb. zeigt allgemein eine erhöhte Tendenz, daktylische Formen zu trochäischen zu reduzieren, dies im Allegro aber noch häufiger als im Lento. Bei den flektierten Adjektiven erreichen sogar 9 von 12 Fällen Werte von über 76 \% (4 davon über $93 \%$ ). Die lb. flektierten Adjektive weisen also in der gesprochenen Sprache in deutlich mehr als der Hälfte der Fälle trochäische oder um eine Silbe reduzierte Formen auf. Nur in 2 Fällen (oppenen und hellegen) zeigen sich mehr nicht-elidierte als elidierte Formen.

\begin{tabular}{|c|c|c|c|}
\hline Wort & reduziert (in \%) & nicht-reduziert (in \%) & unklar (in \%) \\
\hline dréchenen (3) & $87(62)$ & $10(29)$ & $3(9)$ \\
\hline bëllegen (3) & $0(0)$ & $100(100)$ & $0(0)$ \\
\hline kriddelegen (4) & $93(87)$ & $0(7)$ & $7(7)$ \\
\hline edelen (3) & $97(84)$ & $0(9)$ & $3(7)$ \\
\hline adelegen (4) & $87(93)$ & $10(5)$ & $3(2)$ \\
\hline oppenen (3) & $40(22)$ & $43(70)$ & $17(8)$ \\
\hline niddereger (4) & $100(85)$ & $0(3)$ & $0(12)$ \\
\hline monteren (3) & $89(48)$ & $4(48)$ & $7(4)$ \\
\hline sen'sibelen (3) & $93(72)$ & $0(17)$ & $7(12)$ \\
\hline ver'loossenen (3) & 76 & 14 & 10 \\
\hline hellegen (3) & 25 & 75 & 0 \\
\hline eekeleg $(3)^{41}$ & 87 (77) & $3(17)$ & $10(7)$ \\
\hline Gesamt & $71 \%(63 \%)$ & $23 \%(30 \%)$ & $5 \%(7 \%)$ \\
\hline
\end{tabular}

Tabelle 13: Prosodische Realisierung der daktylischen/mehrsilbigen Formen von flektierten Adjektiven (Gruppe (2), in \%) im Allegro. Gesamtzahl (Allegro + Lento) in Klammern, falls zutreffend

\subsubsection{Ergebnisse Verben (Infinitiv)}

Auch die Werte der daktylischen Verben im Infinitiv (Tabelle 14) entsprechen im Groben denen der Gruppen (1a), (1b) und (2): Mit 64 \% zeigen sich in mehr als der Hälfte der Fälle trochäische Formen. Die trochäischen Werte bleiben bei Hinzuziehung der Lento-Werte im Schnitt fast gleich, die daktylischen Werte steigen hingegen (bei weniger unklaren Formen). ${ }^{42}$ Wird ein Verb im Infinitiv mündlich geäußert, tritt den Daten in Tabelle 13 zufolge in der Mehrzahl der Fälle die trochäische Form auf.

\footnotetext{
41 dréchenen ,trockener', bëllegen ,billiger', kriddelegen ,wählerisch', edelen ,edeler', adelegen ,adliger', oppenen ,offener', niddereger ,niedriger', monteren ,munterer', sen 'sibelen ,sensibler', ver'loossenen ,verlassener', hellegen ,heiliger', eekeleg, eklig'.

42 Die auffällig geringen Elisionswerte bei knabberen könnten darauf zurückzuführen sein, dass das Verb am Ende eines Satzes auftrat (D'Maus ass esou aarm drun, si huet keen eenzegen Halm fir drun ze knabberen. ,Die Maus ist so arm dran, sie hat keinen einzigen Halm um dran zu knabbern.').
} 


\begin{tabular}{|c|c|c|c|}
\hline Wort & Trochäus (in \%) & Daktylus (in \%) & unklar (in \%) \\
\hline wanderen (3) & $90(45)$ & $7(53)$ & $3(2)$ \\
\hline 'otemen (3) & $80(87)$ & $3(3)$ & $17(10)$ \\
\hline trëppelen (3) & $93(53)$ & $0(40)$ & $7(7)$ \\
\hline ver'léieren (3) & 30 & 27 & 43 \\
\hline knabberen $(3)^{43}$ & 25 & 60 & 15 \\
\hline Gesamt & $64 \%(62)$ & $19 \%(32)$ & $17 \%(6)$ \\
\hline
\end{tabular}

Tabelle 14: Prosodische Realisierung der daktylischen Formen von Verben im Infinitiv (Gruppe (3), in \%) im Allegro. Gesamtzahl (Allegro + Lento) in Klammern, falls zutreffend

\subsubsection{Zusammenfassung der Ergebnisse}

Trotz großer Unterschiede zwischen den einzelnen Wörtern zeigt sich für die gesprochene lb. Sprache insgesamt eine erhöhte Anzahl an Trochäen gegenüber den ebenfalls möglichen Daktylen. Die Unterschiede zwischen den einzelnen Wortarten sind bis auf die erhöhten Elisionswerte bei den Adjektiven nicht sehr groß. Es gibt also keinen direkten Zusammenhang zwischen Elision und Wortart; die Elision greift wortartunabhängig. Dabei entstehen - vermehrt im Allegro, aber auch im Lento - durch den Silbenausfall häufig aus Daktylen Trochäen. Die oben besprochenen Daten legen daher den Schluss nahe, den Trochäus als die im gesprochenen Lb. umarkierte Fußstruktur zu bezeichnen - im Gegensatz zum in der Schriftsprache dominanten Daktylus. Die Daten erlauben es mit Durchschnittswerten unter $75 \%$ (bis auf wenige Ausnahmen) meines Erachtens allerdings nicht, von einer „kanonischen“ trochäischen Fußstruktur im Lb. zu sprechen, wie dies im Dt. der Fall ist.

Die Wortprosodie der einzelnen lb. Wortarten, wie sie unter 6.1-6.4 gezeichnet wurde, soll abschließend den neuen Ergebnissen angepasst werden. Hierfür werden die Tabellen 7-10 ergänzt und zur besseren Übersicht in Tabelle 15 zusammengefügt.

\footnotetext{
43 wanderen ,wandern', 'otemen ,einatmen', trëppelen ,spazieren gehen', ver'léieren ,verlieren', knabberen
} ,knabbern'. 


\begin{tabular}{|c|c|c|c|c|c|c|c|c|}
\hline \multicolumn{9}{|l|}{ Substantive } \\
\hline Gruppe & \multicolumn{2}{|l|}{$\begin{array}{c}\text { (1a) } \\
\text { Hues,Hase }\end{array}$} & \multicolumn{2}{|c|}{$\begin{array}{c}\text { (1b) } \\
\text { Eemer ,Eimer }\end{array}$} & \multicolumn{2}{|c|}{$\begin{array}{c}\text { (1c) } \\
\text { Bier, Bär }\end{array}$} & \multicolumn{2}{|c|}{$\begin{array}{c}(2) \\
\text { Echo ,Echo' }\end{array}$} \\
\hline \multicolumn{9}{|l|}{ Singular } \\
\hline & Einsilbig & \multicolumn{3}{|c|}{ Trochäus } & \multicolumn{2}{|c|}{ Einsilbig } & \multicolumn{2}{|c|}{ Trochäus } \\
\hline \multicolumn{9}{|l|}{ Plural } \\
\hline & $\begin{array}{l}\text { Trochäus } \\
\text { /Einsilbig }\end{array}$ & \multicolumn{3}{|c|}{$\begin{array}{l}\text { Trochäus } \\
\text { (/Daktylus) }\end{array}$} & \multicolumn{2}{|c|}{$\begin{array}{l}\text { Trochäus } \\
\text { /Einsilbig }\end{array}$} & \multicolumn{2}{|c|}{ Daktylus } \\
\hline \multicolumn{9}{|l|}{ Verben } \\
\hline Gruppe & \multicolumn{2}{|l|}{\begin{tabular}{|l|} 
einsilbiger \\
Stamm (1) \\
\end{tabular}} & \multicolumn{3}{|c|}{$\begin{array}{l}\text { Schwa-Silbe (im } \\
\text { Stamm) (2) }\end{array}$} & \multicolumn{3}{|c|}{$\begin{array}{l}\text { (nicht-silb.) Nasal im } \\
\text { Stammauslaut (3) }\end{array}$} \\
\hline Beispiel & \multicolumn{2}{|c|}{ kucken ,kucken' } & \multicolumn{3}{|c|}{ seegelen , segeln' } & \multicolumn{3}{|c|}{ zeechnen ,zeichnen' } \\
\hline \multicolumn{9}{|l|}{ Infinitiv } \\
\hline & \multicolumn{2}{|l|}{ Trochäus } & \multicolumn{3}{|c|}{$\begin{array}{l}\text { Trochäus } \\
\text { (/Daktylus) }\end{array}$} & \multicolumn{3}{|c|}{$\begin{array}{l}\text { Trochäus } \\
\text { (/Daktylus)(selten) }\end{array}$} \\
\hline \multicolumn{9}{|l|}{ Präsens } \\
\hline & \multicolumn{2}{|l|}{$\begin{array}{l}\text { Trochäus } \\
\text { /Einsilbig }\end{array}$} & \multicolumn{3}{|c|}{$\begin{array}{l}\text { Trochäus } \\
\text { (/Daktylus) }\end{array}$} & \multicolumn{3}{|c|}{ Trochäus } \\
\hline \multicolumn{9}{|l|}{ Adjektive } \\
\hline Grundform & einsilbig & \multicolumn{2}{|c|}{$\begin{array}{l}\text { zweisilbig } \\
\text { auf }-e\end{array}$} & \multicolumn{2}{|c|}{$\begin{array}{l}\text { zweisilbig } \\
\text { auf -el }\end{array}$} & \multicolumn{2}{|c|}{$\begin{array}{l}\text { zweisilbig } \\
\text { auf -en }\end{array}$} & $\begin{array}{l}\text { zweisilbig auf } \\
-e r \\
\end{array}$ \\
\hline Beispiel & $\begin{array}{l}\text { schéin } \\
\text { schön }\end{array}$ & \multicolumn{2}{|c|}{$\begin{array}{l}\text { midd } \\
\text {, müde }\end{array}$} & \multicolumn{2}{|c|}{ eitel , eitel } & \multicolumn{2}{|c|}{ oppen, offen' } & $\begin{array}{l}\text { monter, mun- } \\
\text { ter }\end{array}$ \\
\hline Flektierte Fc & m (silbisches & $10 \mathrm{~A}$ & & & & & & \\
\hline & $\begin{array}{l}\text { Einsilbig/ } \\
\text { Trochäus }\end{array}$ & & & $\begin{array}{l}\text { Trochä } \\
\text { (/Dakty }\end{array}$ & & $\begin{array}{l}\text { Troc } \\
(/ D a\end{array}$ & & $\begin{array}{l}\text { Trochäus } \\
\text { (/Daktylus) }\end{array}$ \\
\hline
\end{tabular}

Tabelle 15: Den Ergebnissen vom Experiment angepasste prosodische Struktur der lb. Substantive, Adjektive und Verben mit prototypischen Beispielen. Die hell unterlegten Flächen zeigen Unterschiede zu den Tabellen 6-9 auf. Die Formen in Klammern stellen die markierten Formen dar. Nicht-trochäische Fußtypen stehen zur besseren Abhebung kursiv

Der wesentliche Unterschied zu den Tabellen 6-9 besteht darin, dass der für die Schriftsprache häufige Daktylus in der gesprochenen Sprache in vielen Fällen dem Trochäus weicht. Da letzterer häufiger auftritt, erscheint er in der angepassten Übersicht ohne Klammern und wird damit als unmarkierte Form betrachtet. Der Daktylus in Klammern stellt in der gesprochenen Sprache die markierte Realisierung dar.

Insgesamt steht die $\mathrm{lb}$. Wortprosodie in ihrer Fußstruktur der dt. nach Auswertung der Sprachdaten näher, als bislang angenommen wurde. Interessant ist hierbei - und hierin liegt auch einer der großen Unterschiede -, dass die trochäische Form im Lb. durch Elision im Stamm entsteht, und dies in allen behandelten Wortarten. Der Schwa-Vokal ist im Lb. im Stamm also weniger stabil als in der Endung. Im Dt. trifft dies nur auf die Adjektive zu, bei den Verben wird der Trochäus durch Schwa-Synkope in der Endung erreicht. Entsprechend greifen in beiden Sprachen bei der Herstellung des unmarkierten Trochäus zum Teil andere Mechanismen. 
Die unter 6.2 getroffene Feststellung, dass im lb. Plural die Morphologie (Pluralmarker) die Prosodie (einheitliche Fußbildung) begrenzt, muss im Hinblick auf die Ergebnisse des Sprachexperiments relativiert werden. Es erscheint nun vielmehr so, dass Morphologie und Prosodie im Plural zusammenspielen: Der Pluralmarker wird bewahrt, zusätzlich steht als prosodisches Mittel zur Trochäenbildung die Tilgung des Schwa-Vokals zwischen der Pluralendung und der (betonten) ersten Silbe zur Verfügung.

Was schließlich die Bestätigung/Falsifizierung von Hyp. 3 (Die Schwa-Elision ist prosodisch gesteuert und dient der Erlangung einer kanonischen trochäischen Fußstruktur) betrifft, ist eine eindeutige Antwort nur schwer möglich. Da die Häufigkeit des Schwa-Ausfalls sich von Wort zu Wort stark unterscheiden kann, ist davon auszugehen, dass er nicht primär das Ziel hat, zu einer kanonischen Fußstruktur beizutragen. Das Ergebnis stellt allerdings durchaus eine Veränderung der prosodischen Struktur dar. Somit muss meines Erachtens die TrochäusBildung als ein Produkt der Schwa-Elision, nicht aber als Ziel des Phänomens angesehen werden. 44

\section{$7 \quad$ Zusammenfassung und Schlussfolgerung}

Die vorliegende Untersuchung befasst sich mit der Elision des Schwa-Vokals im Lb. In Silben, die einer betonten Silbe unmittelbar folgen, und in der Umgebung von Sonoranten $(l, r$, $m, n)$ kann der Vokal getilgt werden, wodurch sich das Wort um eine Silbe verkürzt. Das Phänomen wurde zunächst im Vergleich mit dem Dt. (und dem Ndl.) beschrieben. Im Anschluss wurden drei aus diesen Darstellungen abgeleitete Hypothesen anhand neuer Sprachdaten von 15 SprecherInnen aus dem zentrallb. Dialektraum überprüft.

Hypothese 1 (Erhöhte Sprechgeschwindigkeit begünstigt die Schwa-Elision im Luxemburgischen) kann bestätigt werden. Die für das Lb. allgemein häufige Schwa-Elision trat im Allegro $(64 \%$ der möglichen Fälle) signifikant öfter auf als im Lento $(40 \%)(p<0,001)$. Der Unterschied zwischen den beiden Redegeschwindigkeiten variierte interindividuell zwar stark, die Hypothese traf aber auf alle SprecherInnen zu, unabhängig von Alter und Geschlecht. Eine schnellere Redeweise begünstigt also die Elision des Schwa-Vokals.

Auch Hypothese 2 (Bestimmte Lautverbindungen begünstigen die Schwa-Elision im Luxemburgischen) wurde bestätigt. Schwa-Elision war zwar in allen untersuchten Lautkontexten möglich, einige Kontexte zeigten aber wesentlich höhere Zahlen als andere. So findet die Elision häufig in der Lautverbindung Plosiv/Frikativ + o + Sonorant statt, jedoch weniger häufig, wenn vor Schwa ein Sonorant oder ein Diphthong steht. Einige Lautverbindungen erleichtern den Ausfall, andere erschweren ihn. Hierbei wurde auch die Sonorität der Schwa umgebenden Konsonanten als wichtiger Faktor angeführt.

Hypothese 3 (Die Schwa-Elision ist prosodisch gesteuert und dient der Erlangung einer kanonischen trochäischen Fußstruktur) stellt den Kern der Untersuchung dar. Eine Gegenüberstellung der dt. und lb. Wortprosodie der Wortarten Substantiv, Adjektiv und Verben zeigte, dass im Lb. neben dem Trochäus auch Einsilbler und häufig Daktylen vorkommen können. Im Dt.

\footnotetext{
${ }^{44}$ Die Hinzuziehung von Komposita, weiterer Verbformen - etwa des Partizip Perfekts -, der Komparationsformen der Adjektive sowie - in größerem Maße - Fremdwörtern bleibt zunächst ein Forschungsdesiderat. Entsprechende Ergebnisse können diejenigen dieser Untersuchung jedoch sicherlich fruchtbar ergänzen.
}

ISSN 1615-3014 
hingegen dominiert der Trochäus. Nach Analyse der Sprachdaten aus dem Sprachexperiment ergab sich jedoch, dass im Lb. in allen untersuchten Wortarten die daktylische Form durch Ausfall des Schwa-Vokals in (deutlich) mehr als der Hälfte der Fälle von der trochäischen Form abgelöst wird. Durch Schwa-Elision verschiebt sich somit die prosodische Struktur zugunsten des Trochäus. Wie im Dt. herrscht der Trochäus im Lb. also vor. Anders als im Dt. treten jedoch zusätzlich weiterhin zahlreiche Daktylen auf. Daneben liegen der Trochäenbildung mit Schwa-Ausfall im Stamm (und nicht in der Endsilbe) in beiden Sprachen zum Teil andere Mechanismen zugrunde. Hyp. 3 kann aufgrund der großen wortspezifischen Variation dennoch nicht bestätigt werden: Die Veränderung der prosodischen Fußstruktur erscheint als Produkt, nicht aber als Ziel der Schwa-Elision. Sie hat große Konsequenzen für die Wortprosodie, ist aber nicht primär prosodisch gesteuert.

Insgesamt erscheint die Schwa-Elision im Lb. als ein Phänomen, das aus verschiedenen Perspektiven beschrieben und nur durch deren Kombination erklärt werden kann: Eine schnellere Redegeschwindigkeit hebt die Elisionswahrscheinlichkeit (realisationsphonetischer Aspekt); der Erhalt bzw. Ausfall des Schwa-Vokals hängt von der direkten phonologischen Umgebung ab (artikulatorischer Aspekt); als Auswirkung der Schwa-Elision, nicht aber als Begründung, ergibt sich eine Veränderung der Wortprosodie (der prosodische Aspekt).

Offen bleiben Fragen nach der Elisionsmöglichkeit anderer Vokale als dem Schwa-Vokal, der Häufigkeit der Elision bei mehrsilbigen Wörtern (Typ: Om(e)letten, siehe Auswertung oben), der jeweiligen Resilbifizierung der durch Wegfall des Schwa-Nukleus frei gewordenen Konsonanten sowie der Elisionswahrscheinlichkeit des Schwa-Vokals in der Umgebung bestimmter einzelner Laute (etwa $k+\jmath+l$ ). Interessant ist auch die Frage nach dem Einfluss der Worthäufigkeit oder die nach der (privaten) Schriftlichkeit (wird der Vokal, etwa in der privaten E-Mail-Korrespondenz, geschrieben oder nicht?). Die Schwa-Elision im Lb. bietet also noch jede Menge interessanter Aspekte, die eingehender erforscht werden können.

\section{Literatur}

Booij, Gert (1995): The phonology of Dutch. Oxford: Oxford University Press.

Braun, Josy et al. (2005): Les verbes luxembourgeois. D'Lëtzebuerger Verben. Mamer: Guy Binsfeld.

Conrad, François (2010): Das Luxemburgische Schwa. Zentraler Vokal einer jungen germanischen Sprache. Unveröffentlichte Magisterarbeit, Universitäten Bamberg und Luxemburg.

Dressler, Wolfgang Ulrich (1972a): Allegroregeln rechtfertigen Lentoregeln. Sekundäre Phoneme des Bretonischen. Innsbruck: Institut für Vergleichende Sprachwissenschaft der Universität Innsbruck (= Innsbrucker Beiträge zur Sprachwissenschaft 9).

Dressler, Wolfgang Ulrich (1972b): „Phonologische Schnellsprechregeln in der Wiener Umgangssprache“. Wiener Linguistische Gazette 1: 1-29.

Eisenberg, Peter (1991): „Syllabische Struktur und Wortakzent. Prinzipien der Prosodik deutscher Wörter". Zeitschrift für Sprachwissenschaft 10/1: 37-64.

Féry, Caroline (1991): "German schwa in prosodic morphology". Zeitschrift für Sprachwissenschaft 10/1: 65-85.

Gilles, Peter (1998): „Die Emanzipation des Lëtzebuergeschen aus dem Gefüge der deutschen Mundarten“. In: Besch, Werner/Solms, Hans Joachim (eds.): Regionale Sprachgeschichte. Zeitschrift für Deutsche Philologie 117 (Sonderheft): 20-35.

ISSN 1615-3014 
Gilles, Peter (1999): Dialektausgleich im Lëtzebuergeschen. Zur phonetisch-phonologischen Fokussierung einer Nationalsprache. Tübingen: Max Niemeyer. (= Phonai 44).

Gilles, Peter (2010): „Wie Französisch, nur Ton 1. Der Wortakzent des Deutschen und Luxemburgischen im Kontrast“. In: Dammel, Antje/Kürschner, Sebastian/Nübling, Damaris (eds.): Kontrastive Germanistische Linguistik. Bd. 1. Hildesheim, Georg Olms: 109-140.

Gilles, Peter/Trouvain, Jürgen (2013): "Luxembourgish". Journal of the International Phonetic Association 43/1: 67-74.

Gilles, Peter (2014): "Phonological domains in Luxembourgish and their relevance for the phonological system". In: Caro Reina, Javier/Szczepaniak, Renata (eds.): Phonological Typology of Syllable and Word languages in Theory and Practice. Berlin/New York, de Gruyter: 279-304.

Hall, Tracy Alan (2000): Phonologie. Eine Einführung. Berlin/New York: de Gruyter.

Hooper, Joan (1978): “Constraints on schwa-deletion in American English". In: Fisiak, Jacek (ed.): Recent Developments in historical Phonology. The Hague, Mouton: 183-207.

Kohler, Klaus (1979): „Kommunikative Aspekte satzphonetischer Prozesse im Deutschen“. In: Vater, Heinz (ed.): Phonologische Probleme des Deutschen. Tübingen, Stauffenburg: 13-39.

Kohler, Klaus (1990): "Segmental reduction in connected speech in German: Phonological facts and phonetic explanations”. In: Hardcastle, William J./Marchal, Alain (eds.): Speech production and speech modeling. Dordrecht, Kluwer: 69-92.

Kohler, Klaus (1995): Einführung in die Phonetik des Deutschen. 2. Auflage. Berlin: Erich Schmidt. (= Grundlagen der Germanistik 20).

Krier, Fernande (2008): "Réductions et amplifications phonétiques en luxembourgeois ». In: Stehl, Thomas: Kenntnis und Wandel der Sprachen. Beiträge zur Potsdamer Ehrenpromotion für Helmut Lüdtke. Tübingen, Narr: 93-107. (= Tübinger Beiträge zur Linguistik 507).

Meinhold, Gottfried/Stock, Eberhard (1982): Phonologie der deutschen Gegenwartssprache. 2. Auflage. Leipzig: Bibliographisches Institut.

Moulin, Claudine/Nübling, Damaris (eds.) (2006): Perspektiven einer linguistischen Luxemburgistik. Heidelberg: Universitätsverlag Winter.

Nübling, Damaris (2005): „Das Lëtzebuergesche als Herausforderung für die Linguistik“. In: Lëtzebuergesch - Entwicklungstendenzen und Forschungsperspektiven einer jungen Sprache. Beiträge zum Workshop Lëtzebuergesch, November 2001 - Luxemburg/Mersch. Institut Grand-Ducal. Section de Linguistique, d'Ethnologie et d'Onomastique/Centre National de Littérature. Luxemburg/Mersch: 147-168.

Pompino-Marschall, Bernd (2009): Einführung in die Phonetik. 3. Auflage. Berlin/New York: de Gruyter.

Schanen, François/Zimmer, Jacqui (2005): 1, 2, 3 Lëtzebuergesch Grammaire (1). Le groupe verbal. Esch-sur-Alzette: Schortgen.

Schanen, François/Zimmer, Jacqui (2006a): 1, 2, 3 Lëtzebuergesch Grammaire (2). Le groupe nominal. Esch-sur-Alzette: Schortgen.

Schanen, François/Zimmer, Jacqui (2006b): 1, 2, 3 Lëtzebuergesch Grammaire (3). L'orthographe. Esch-sur-Alzette: Schortgen.

Schanen, Francois (2006): " Prosodie luxembourgeoise ». In: Craemer, Susanne et al. (eds.): Europäische Begegnungen. Beiträge zur Literatur-wissenschaft, Sprache und Philosophie. Festschrift für Joseph Kohnen. Luxemburg, Universität Luxemburg: 509-522. 
Szczepaniak, Renata (2010): „Die phonologisch-typologische Entwicklung des Deutschen und des Luxemburgischen im Kontrast“". In: Dammel, Antje/Kürschner, Sebastian/Nübling, Damaris (eds.): Kontrastive Germanistische Linguistik, Bd. 2. Hildesheim, Georg Olms: 85-110.

\section{Anhang a: Die Wörter des Sprachexperiments}

Die Punkte (1)-(3) listen die Wörter der einzelnen Sprechgeschwindigkeiten auf. Eine „2“ bezeichnet Zweifachnennung, ,allegro“ besagt, dass die doppelte Form nur im Allegro-Teil, „lento“, dass die doppelte Form nur im Lento-Teil auftrat. (en) zeigt an, dass das Wort einmal in seiner Singular- und einmal in seiner Plural-Form auftrat. Die Formen in der Übersicht stellen stets die Maximalformen (ohne Elision) dar. Flexion und Numerus entsprechen den Formen, wie sie im Satzzusammenhang oder in den Wortketten im Experiment auftraten. Die Zahl in Klammern bezeichnet die Anzahl der Wörter pro Punkt. Die Übersetzung der Wörter befindet sich in der jeweiligen Fußnote. Der elisionsfähige Vokal ist unterstrichen.

1. Wörter, die sowohl im Lento als auch im Allegro auftraten (33) $)^{45}$

Trommeler, Baueren, Ministeren, Konferenz, Temperatur(en) (2/allegro), Eemeren, Duechteren, Éisterräich, wann-ech-gelift, Prabbeli(en) (2/lento), dréchenen, bëllegen (2/lento), liberalen, bastelen, kriddelegen, Omelett(en) (2/lento), schokelas (2/allegro), eekeleg, wanderren,

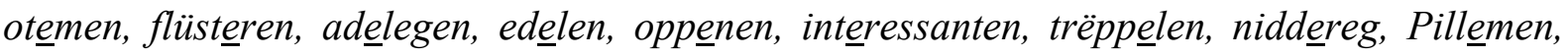
monteren, sensibelen

\footnotetext{
${ }^{45}$ Trommeler ,Trommler', Baueren ,Bauern', Ministeren ,Minister', Konferenz ,Konferenz', Temperatur(en) ,Temperatur(en)', Eemeren ,Eimer (Pl) ${ }^{6}$, Duechteren ,Töchter ${ }^{6}$, Éisterräich ,Österreich', wann-ech-gelift ,bitte', Prabbeli(en) ,Regenschirm(e)', dréchenen ,trockener', bëllegen ,billiger', liberalen ,liberaler', bastelen ,bastelen', kriddelegen ,wählerisch', Omelett(en) ,Omelett(s)', schokelas ,aus Schokolade', eekeleg ,eklig', wanderen ,wandern', otemen ,atmen', flüsteren ,flüstern', adelegen ,adliger', edelen ,edeler', oppenen ,offener', interessanten ,interessanter', trëppelen ,spazieren gehen', niddereg ,niedrig', Pillemen ,Kissen', monteren ,munterer', sensibelen ,sensibler'
} 
2. Wörter, die nur im Lento auftraten (3) 46

traueregen, néierens, hall(e)wen ${ }^{47}$

3. Wörter, die nur im Allegro auftraten $(21)^{48}$

Elteren, wat fịr eng, verloossener, Gromperen, hellegen, Ieselen, Brauerei, Seegelen, Seegeler, Zigarett(en) (2/allegro), Restauranten, Ouschteren, houfereg, verléieren, Aueren, origineller, Aangeler, ronderëm, Interessi, knabberen

\section{Anhang b: Beispiel-Stimuli aus dem Experiment}

Die Punkte (1) und (2) zeigen jeweils ein Beispiel-Stimulus im Allegro und im Lento. Die Testpersonen sollten die Sätze anhand der jeweiligen Bilder vervollständigen (ein paar Wörter sollten aus dem Französischen ins Luxemburgische übersetzt werden) und in der vorgegebenen Redegeschwindigkeit zweimal vorlesen.

1. Beispiel-Stimulus Allegro:

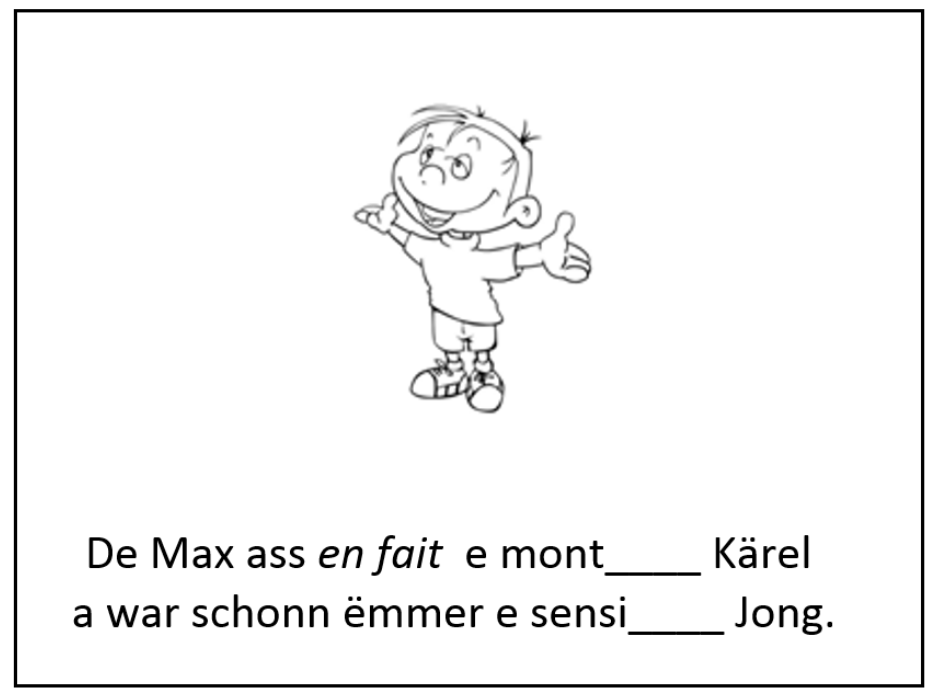

Der Satz in der Form, wie er von den Testpersonen vorgelesen wurde, lautet (die ausgefüllten bzw. übersetzten Wörter oder Wortteile sind unterstrichen):

De Max ass eigentlech e montere Kärel a war schonn ëmmer e sensibele Jong. ,Max ist eigentlich ein munterer Kerl und war schon immer ein sensibler Junge.

\footnotetext{
46 traueregen ,trauriger', néierens ,nirgends', hall(e)wen ,halber“

47 Das Adjektiv hallwen trat in den Wörterbüchern stets ohne Schwa auf, wurde zur Überprüfung dennoch ins Experiment eingebaut. Es bestätigte sich die schwa-lose Form der Wörterbucheinträge.

${ }^{48}$ Elteren ,Eltern', wat fir eng ,was für eine', verloossener ,verlassener', Gromperen ,Kartoffeln', hellegen ,heiliger‘, Ieselen ,Esel‘, Brauerei „Brauerei‘, Seegelen ,Segel (Pl.)‘, Seegeler ,Segler‘, Zigarett(en) ,Zigarett(en)', Restauranten ,Restaurants', Ouschteren ,Ostern', houfreg ,stolz', verléieren ,verlieren', Aueren ,Uhren', origineller ,origineller', Aangeler ,Angler', ronderëm ,rundherum‘, Interessi ,Interesse', knabberen ,knabbern"
} 
2. Beispiel-Stimulus Lento:

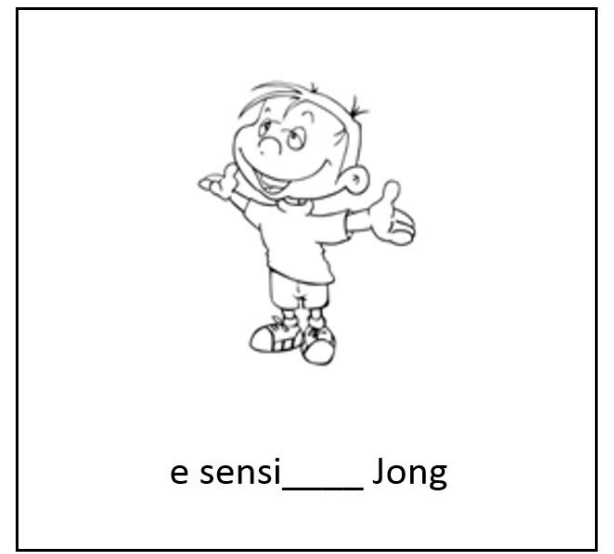

Der Satzteil in der Form, wie er von den Testpersonen vorgelesen wurde, lautet (der ausgefüllte Wortteil ist unterstrichen):

e sensibele Jong, ein sensibler Junge 